Alma Mater Studiorum - Università di Bologna DEPARTMENT OF ECONOMICS

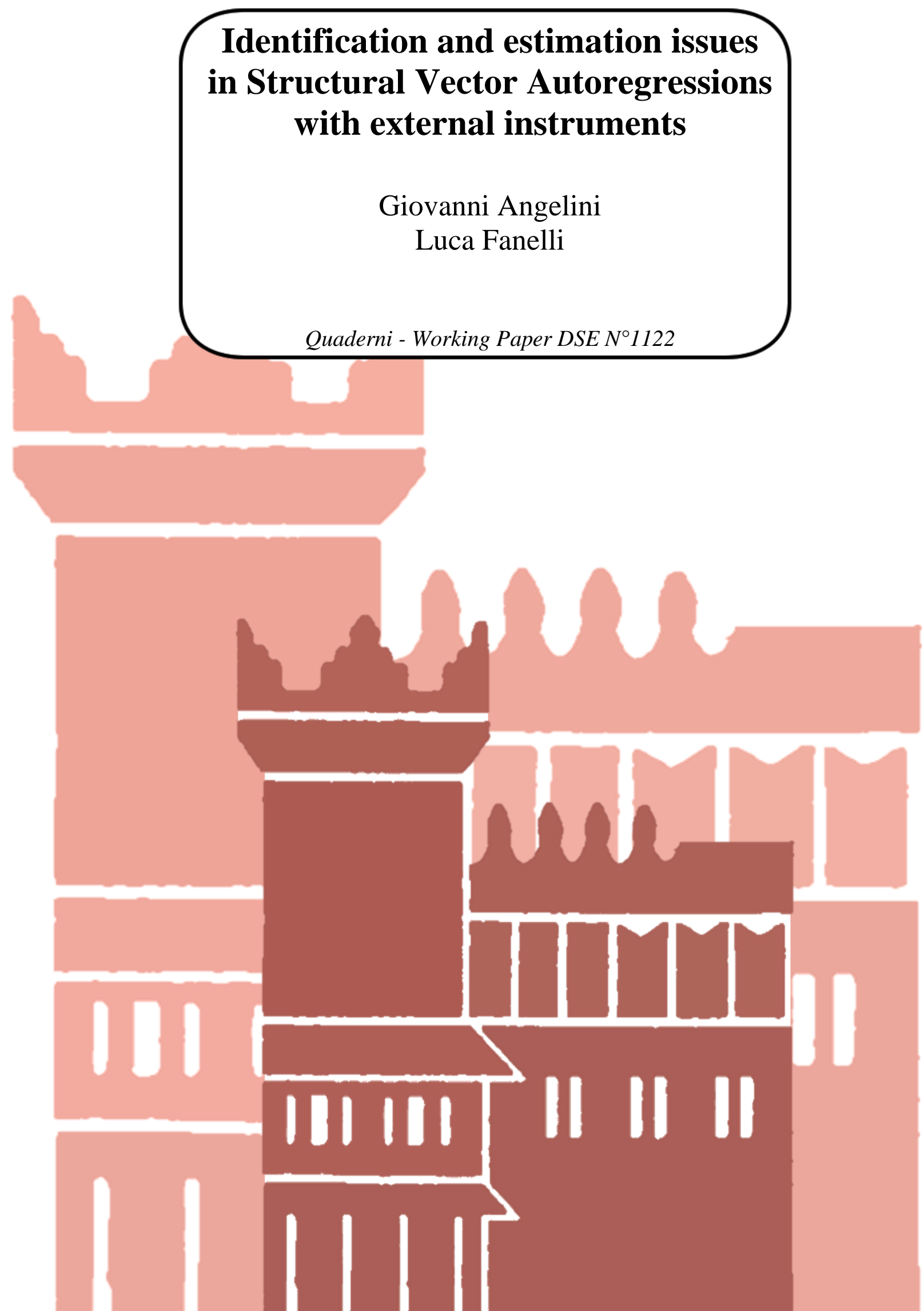




\title{
Identification and estimation issues in Structural Vector Autoregressions with external instruments
}

\author{
Giovanni Angelini, Luca Fanelli* \\ Department of Economics, University of Bologna
}

May 2018

\begin{abstract}
In this paper we discuss general identification results for Structural Vector Autoregressions (SVARs) with external instruments, considering the case in which $r$ valid instruments are used to identify $g \geq 1$ structural shocks, where $r \geq g$. We endow the SVAR with an auxiliary statistical model for the external instruments which is a system of reduced form equations. The SVAR and the auxiliary model for the external instruments jointly form a 'larger' SVAR characterized by a particularly restricted parametric structure, and are connected by the covariance matrix of their disturbances which incorporates the 'relevance' and 'exogeneity' conditions. We discuss identification results and likelihood-based estimation methods both in the 'multiple shocks' approach, where all structural shocks are of interest, and in the 'partial shock' approach, where only a subset of the structural shocks is of interest. Overidentified SVARs with external instruments can be easily tested in our setup. The suggested method is applied to investigate empirically whether commonly employed measures of macroeconomic and financial uncertainty respond on-impact, other than with lags, to business cycle fluctuations in the U.S. in the period after the Global Financial Crisis. To do so, we employ two external instruments to identify the real economic activity shock in a partial shock approach.
\end{abstract}

Keywords: External Instruments, Identification, Maximum Likelihood, SVARs, Uncertainty.

J.E.L.: C32, C51, E44, G10.

${ }^{*}$ Corresponding author: Department of Economics, University of Bologna, Piazza Scaravilli 2, I-40126 Bologna, luca.fanelli@unibo.it 


\section{Non-technical Summary}

This paper deals with Structural Vector Autoregressions (SVARs). SVARs are largely used in applied macroeconomics to capture stylized empirical facts, shock transmission mechanisms and dynamic causal effects. In order to identify the shocks, it is necessary to place restrictions (typically, but not necessarily, zero restrictions) on the 'matrix of structural parameters' which is the matrix which maps the structural shocks onto the reduced form VAR disturbances. One of the most interesting approaches developed recently to identify structural shocks by possibly avoiding recursive structures or direct implausible restrictions on the matrix of structural parameters is the so-called 'external instruments' or 'proxy-SVAR' approach of Stock and Watson (2008, 2012, 2018) and Mertens and Ravn (2013, 2014). This method takes advantage of information developed from 'outside' the VAR in the form of variables which are correlated with the latent structural shocks of interest and are uncorrelated with the other structural shocks of the system. The external instruments provide additional moment conditions which can be used along with the standard covariance restrictions implied by SVARs to identify the shocks.

The emerging literature on proxy-SVARs is mainly focused on the use of one external instrument to identify a single structural shock of interest in isolation from all the other shocks of the system. For example, Stock and Watson (2012) identify six shocks (the oil shock, the monetary policy shock, the productivity shock, the uncertainty shock, the liquidity/financial risk shock and the fiscal policy shock) by exploit many external instruments, but use then one at the time; see also Ramey (2016). In general, there exists no general result in the literature which provides a guidance for practitioners to address the following question: given $g \geq 1$ structural shocks

of interest and $r \geq g$ external instruments for these shocks, how many restrictions do we need for the model to be identified, and where do these restrictions need to be placed? The paper provides such a general framework and complements the analysis of proxy-SVARs with novel likelihood-based estimation methods.

The suggested approach takes a stand on the process which generates the external instruments. This is an auxiliary model which is specified as a dynamic reduced form whose disturbances incorporate the "relevance" condition, i.e. the correlation with the structural shocks of interest, and the "exogeneity" condition, i.e. the absence of correlation with the other structural shocks of the system. The SVAR and the specified auxiliary model for the instruments form a "larger" SVAR which is called AC-SVAR model, where "AC" stands for "augmentedconstrained". The AC-SVAR is "augmented" because it is obtained by adding the auxiliary statistical model for the instruments to the SVAR. The AC-SVAR is "constrained" because it is characterized by a particular triangular structure in the autoregressive coefficients and a particular constrained structure in the matrix which maps the reduced form disturbances to the 
structural shocks.

The AC-SVAR model allows to generalize the analysis of proxy-SVARs to the setup $r \geq g \geq$ 1. Two cases are considered: the "multiple shocks" approach, where all structural shocks are of interest (including the non-instrumented ones), and the "partial shock" approach, where only the g instrumented structural shocks are of interest. Novel identification results and likelihood-based estimation methods are discussed for these two cases.

The AC-SVAR methodology is applied to U.S. monthly data by considering a small-scale system which includes measures of macroeconomic and financial uncertainty taken from Jurado et al. (2015) and Ludvigson et al. (2018), respectively, and a measure of real economic activity, say the industrial production growth. The objective is to investigate whether the selected measures of macroeconomic and financial uncertainty respond on-impact (instantaneously) to an identified real economic activity shock on the "Great Recession+Slow Recovery" period 20082015. In order to identify the real economic activity shock, two external instruments are jointly employed by using the results discussed in the paper. Estimation results suggest that both macroeconomic and financial uncertainty respond significantly to the identified real economic activity shock one month after the shock, but not on-impact. This evidence enriches an ongoing debate on the role of uncertainty in the business cycle (see Ludvigson et al., 2018). 


\section{Introduction}

Structural Vector Autoregressions (SVARs) provide stylized and parsimonious characterizations of shock transmission mechanisms and allow to track dynamic causality effects. However, the identification of SVARs requires parameter restrictions on the matrix which maps the VAR disturbances to structural shocks, henceforth denoted with $B$, that are often implausible. The parameters in the matrix $B$ capture the on-impact (instantaneous) effect of the structural shocks on the variables, and are also a crucial ingredients of the Impulse Response Functions (IRFs). One of the most interesting approaches developed in the recent literature to identify structural shocks by possibly avoiding recursive structures or direct implausible assumptions on the elements of $B$ is the so-called 'external instruments' or 'proxy-SVAR' approach, see Stock and Watson (2008, 2012, 2018), Mertens and Ravn (2013, 2014); see also Stock (2008). This method takes advantage of information developed from 'outside' the VAR in the form of variables which are correlated with the latent structural shocks of interest, and are uncorrelated with the other structural shocks of the system. Throughout the paper we use the terms 'SVARs with external instruments' and 'proxy-SVARs' interchangeably.

This paper provides a novel contribution on SVARs with external instruments. The emerging literature on proxy-SVARs is mainly focused on the use of one external instrument to identify a single structural shock of interest in isolation from all the other shocks of the system. For example, Stock and Watson (2012) identify six shocks (the oil shock, the monetary policy shock, the productivity shock, the uncertainty shock, the liquidity/financial risk shock and the fiscal policy shock) by exploit many external instruments, but use then one at the time; see also Ramey (2016). In general, there exists no general result in the literature which provides a guidance for practitioners to address the following question: given a $g \geq 1$ structural shocks of interest and $r \geq g$ external instruments available for these shocks, how many restrictions do we need for the model to be identified, and where do these restrictions need to be placed? We provide such a general framework and complement the analysis of proxy-SVARs with novel likelihood-based estimation methods.

Our approach takes a stand on the process which generates the external instruments. This is an auxiliary model which is specified as a dynamic reduced form whose disturbances incorporate the 'relevance' condition, i.e. the correlation with the structural shocks of interest, and the 'exogeneity' condition, i.e. the absence of correlation with the other structural shocks of the

system. We augment the SVAR with the specified auxiliary model for the instruments obtaining a 'larger' SVAR which is called AC-SVAR model, where 'AC' stands for 'augmented-constrained'. The AC-SVAR is 'augmented' because it is obtained by adding the auxiliary statistical model for the instruments to the original SVAR equations. The AC-SVAR is 'constrained' because 
it is characterized by a particular triangular structure in the autoregressive coefficients and a particular constrained structure in the matrix which maps the reduced form disturbances to the structural shocks.

The AC-SVAR model allows to generalize the analysis of proxy-SVARs to the setup $r \geq g \geq$ 1. We focus on two cases: the 'multiple shocks' approach and the 'partial shock' approach. In the former, $r$ external instruments are used for $g$ instrumented structural shocks, but it is possible to infer the dynamic causal effects of all structural shocks of the system, i.e. also the ones of the noninstrumented structural shocks. This implies that all columns of the matrix $B$ can be identified - without imposing direct restrictions on its elements - and IRFs can be computed accordingly. We show that in this case the identification and estimation of the AC-SVAR amounts to the practice of identifying and estimating 'conventional' SVARs by 'internal' restrictions. More precisely, we study the conditions under which the AC-SVAR model is identified, and show that identified models can be estimated by suitably adapting 'standard' maximum likelihood methods 1 The inference on the IRFs of interest can be performed by existing methods, reviewed e.g. in Lütkepohl and Kilian (2017).

In the partial shock approach, $r$ external instruments are used for $g$ instrumented shocks, but now the interest is solely focused on the dynamic causal effects of these $g$ structural shocks regardless of the other structural shocks of the system. This implies that only a subset of $g$ columns of $B$, collected in $B_{1}$, need to be identified in order to compute the IRFs of interest. When $r=g=1, B_{1}$ is a column of $B$ (typically the first column) and the parameter which captures the correlation between the external instrument and the shock of interest is a scalar, say $\phi$. We relax this condition and derive identification and estimation results for the general case $r \geq$ $g \geq 1$, so that $\phi=\Phi$ becomes a matrix with $r$ rows and $g$ columns containing a set of 'relevance' parameters. We show that when in this context the necessary and sufficient rank condition for identification is satisfied, estimation can be carried out by a classical minimum distance (CMD) approach which minimizes the distance between a set of 'reduced form' parameters, which can be easily estimated by Gaussian maximum likelihood from the AC-SVAR model, and a set of parameters which include the non-zero elements of $B_{1}$ and $\Phi$, respectively. Also in this framework, the inference on the IRFs can be performed by standard methods, see e.g. Lütkepohl and Kilian (2017).

To show the empirical usefulness of our approach, we focus on the U.S. economy after the Global Financial Crisis and apply the suggested AC-SVAR methodology to a small-scale monthly system which includes measures of macroeconomic and financial uncertainty taken from Jurado

\footnotetext{
${ }^{1}$ In principle, any existing econometric package which features the estimation of SVARs can potentially be adapted along the lines discussed in the paper, Section 4.
} 
et al (2015) and Ludvigson et al. (2018), respectively, and a measure of real economic activity, say the industrial production growth. Results in e.g. Bloom (2009), Stock and Watson (2012), Jurado et al (2015), Leduc and Liu (2016), Nakata (2017), Caggiano et al. (2017), inter alia, obtained with a variety of identification methods, show that uncertainty explains a sizeable fraction of the decline in real economic activity, especially after 2008. Our objective, however, is different. We focus on the other direction of causality, i.e. on whether the selected measures of macroeconomic and financial uncertainty respond on-impact (i.e. instantaneously) to a real economic activity shock on the 'Great Recession+Slow Recovery' period 2008-2015. Ludvigson et al. (2018) argue that if uncertainty measures responds on-impact to 'first-moment' shocks, then uncertainty can not be considered an exogenous driver of the decline of economic activity, but rather an endogenous response to it. This investigation represents a crucial step towards the understanding of the channels through which uncertainty affects the economy, and allows to discriminate empirically among two classes of models which treat uncertainty either as an exogenous driver of the business cycle, or as an endogenous response to economic fluctuations (Ludvigson et al. 2018). Interestingly, addressing this empirical objective requires the estimation of non-recursive SVARs, i.e. models where the matrix $B$ is non-recursive (non-triangular), which makes the suggested proxy-SVAR approach attractive.

We follow a partial shock approach in which the shock of interest for which we employ valid external instruments is the real economic activity shock and analyze whether macroeconomic and financial uncertainty measures respond on-impact (other than with lags) to the identified real economic activity shock. We regress the variations in hours worked and capacity utilization on the 'price of gold' proxy of uncertainty employed by Piffer and Podstawski (2018), and model the residuals obtained from this regression as two valid external instruments for the real economic activity shock in an AC-SVAR system. These instruments are correlated with the real economic activity shock (relevance), and are expected to be uncorrelated with macroeconomic and financial uncertainty shocks (exogeneity) under the conditions discussed in Piffer and Podstawski (2018). We obtain an overidentified proxy-SVAR which is estimated and not rejected by the data on Great Recession+Slow Recovery' period 2008-2015. Formal tests and IRFs support the hypothesis that macroeconomic and uncertainty measures respond significantly to the identified real economic activity shock one month after the shock, but not on-impact.

This paper is naturally connected with the increasing strand of the macroeconometric literature which develops and estimation and inferential methods for SVARs with external instruments, and apply these methods to various research fields. In addition to the key works cited above, frequentist inferential methods for proxy-VARs are discussed in e.g. Lunsford (2015), Jentsch and Lunsford (2016) and Olea et al. (2016), while Bayesian inferential methods and 
implementations may be found in Caldara and Herbst (2018) and Arias et al. (2018). Important applied developments include e.g. Gertler and Karadi (2015), Carriero et al. (2015) and Caldara and Kamps (2017); see also Hamilton (2003) and Kilian (2008) for early applications. Moreover, the proxy-SVAR approach is often combined with other identification methods as shown in Ludvigson et al. (2017, 2018), Piffer and Podstawski (2018), Braum and Brüggemann (2017) and Plagborg-Møller and Wolf (2018). Compared to this literature, our paper provides a general framework to study the identification of proxy-SVARs and offers, as a by-product, a novel likelihood-based estimation approach. Only a few of papers make explicit use of auxiliary models for the external instruments to identify and make inference in proxy-SVARs. Plagborg-Møller and Wolf (2018) is a remarkable example in the frequentist setup. In the Bayesian framework, Caldara and Herbst (2018) refer to a Bayesian proxy-SVAR (BP-SVAR) which incorporates the external instrument in the system's likelihood and makes efficient use of the information contained in the proxy. However, these authors limit their attention to the case of an instrument and a single structural shock of interest; see also Arias et al. (2018). Another distinguishing feature of our approach is the focus on the overidentification restrictions which arise in the AC-SVAR framework from the interaction between the $r$ external instruments and the $g$ instrumented shocks. Overidentified proxy-SVARs can be easily tested in our setup. We show that the rejection of the overidentification restrictions may be also due to the non exogeneity of the external instruments (which are assumed relevant). Thus, our methodology provides analogs of 'Sargan's specification test' in the instrumental variable framework, or Hansen's J-test in the generalized method of moments framework, i.e. specification tests for the overall validity of the estimated proxy-SVAR. This appears a novelty in the literature on SVARs with external instruments.

As concerns the empirical illustration, the papers closest to our are Ludvigson et al. (2018), Angelini et al. (2017) and Carriero et al. (2018), who also apply small-scale non-recursive SVARs to analyze the exogeneity/endogeneity of uncertainty and report mixed evidence. Ludvigson et al. (2018) use a three-equations SVARs and the same monthly variables we consider, although their analysis is based on the longer period 1960-2015. They combine the use of external instruments with a particular set-identification approach, where properly selected 'event constraints' are directly imposed on the shocks to achieve identification. Conversely, we confine our main attention to the identification of the real economic activity shock and its impact on macroeconomic and financial uncertainty. Carriero et al. (2018) identify the shocks also in a SVAR framework using a stochastic volatility approach by using measures of macroeconomic and financial uncertainty one at a time, not jointly.

The rest of the paper is organized as follows. Section 2 introduces the reference SVAR with 
external instruments and presents the background and the main assumptions. Section 3 discusses the AC-SVAR model. Section 4 deals with the multiple shocks approach; Section 4.1 focuses on identification issues and Section 4.2 on the estimation procedure. Section 5 deals with the partial shock approach; Section 5.1 focuses on identification issues and Section 5.2 on estimation. Section 6 presents the empirical illustration. Section 7 contains some conclusions. Additional technical details and Monte Carlo experiments are confined in a Technical Supplement ? $^{2}$

Throughout the paper we use the following notation, matrices and conventions, most of which are taken from Magnus and Neudecker (1999). Let $M$ be any $n \times g$ matrix. We use $\operatorname{rank}(\cdot)$ to denote the rank of a matrix. $K_{n g}$ is the $n g \times n g$ commutation matrix, i.e. the matrix such that $K_{n g} \operatorname{vec}(M)=\operatorname{vec}\left(M^{\prime}\right)$. When $n=g$, we use $K_{n}$ for $K_{n n}$. Assume now $M$ is $n \times n$. $D_{n}$ is the duplication matrix, i.e. the $n^{2} \times \frac{1}{2} n(n+1)$ full-column rank matrix such that $D_{n} \operatorname{vech}(M)=\operatorname{vec}(M)$, where $\operatorname{vech}(M)$ is the column obtained from $\operatorname{vec}(M)$ by eliminating all supra-diagonal elements. Given $K_{n}$ and $D_{n}, N_{n}:=\frac{1}{2}\left(I_{n^{2}}+K_{n}\right)$ is a $n^{2} \times n^{2}$ matrix such that $\operatorname{rank}\left(N_{n}\right)=\frac{1}{2} n(n+1)$ and $D_{n}^{+}:=\left(D_{n}^{\prime} D_{n}\right)^{-1} D_{n}^{\prime}$ is the Moore-Penrose inverse of $D_{n}$. We also use the notation $M=M(\theta)$ to indicate that the elements of the matrix $M$ depends on the parameters in the vector $\theta$. Given $\theta=\theta_{0}$, we call 'regular' a matrix such that $\operatorname{rank}(M(\theta))=n$ in a neighborhood of $\theta_{0}$.

\section{Model and background}

We start from the SVAR system

$$
Y_{t}=\Pi X_{t}+\Upsilon_{y} D_{y, t}+u_{t}, u_{t}=B \varepsilon_{t}, t=1, \ldots, T
$$

where $Y_{t}$ is the $n \times 1$ vector of endogenous variables, $X_{t}:=\left(Y_{t-1}^{\prime}, \ldots, Y_{t-k}^{\prime},\right)^{\prime}$ is $n k \times 1, \Pi:=$ $\left(\Pi_{1}: \ldots: \Pi_{k}\right)$ is the is $n \times n k$ matrix containing the autoregressive (slope) parameters, $D_{y, t}$ is an $d_{y}$-dimensional vector containing deterministic components (constant, dummies, etc.) with associated parameters contained in the $n \times d_{y}$ matrix $\Upsilon_{y}$; finally, $u_{t}$ is the $n \times 1$ vector of reducedform disturbances with positive definite covariance matrix $\Sigma_{u}:=E\left(u_{t} u_{t}^{\prime}\right)$. The initial conditions $Y_{0}, \ldots, Y_{1-k}$ are treated as fixed. The system of equations $u_{t}=B \varepsilon_{t}$ in eq. (1) maps the $n \times 1$ vector of structural shocks $\varepsilon_{t}$ to the reduced form disturbances through the $n \times n$ matrix $B$. The structural shocks $\varepsilon_{t}$ are assumed White Noise and may have either normalized covariance matrix $E\left(\varepsilon_{t} \varepsilon_{t}^{\prime}\right):=\Sigma_{\varepsilon}:=I_{n}$, or diagonal covariance matrix $\Sigma_{\varepsilon}:=\operatorname{diag}\left(\sigma_{1}^{2}, \ldots, \sigma_{n}^{2}\right)$. In the second case, the link between reduced form disturbances and structural shocks can be expressed in the form $u_{t}=B^{*} \Sigma_{\varepsilon}^{1 / 2} \varepsilon_{t}^{*}$, where $\varepsilon_{t}^{*}:=\Sigma_{\varepsilon}^{-1 / 2} \varepsilon_{t}$ and $B^{*}$ has exactly the same structure as $B$ in eq. (1)

\footnotetext{
${ }^{2}$ Available online at http://www.rimini.unibo.it/fanelli/TS_AngeliniFanelli_ProxySVAR.pdf
} 
except that the elements on the main diagonal are normalized to 1 . Throughout the paper we follow the parametrization based on $u_{t}=B \varepsilon_{t}$ with $\Sigma_{\varepsilon}=I_{n}$, except where indicated.

We call the elements in $\left(\Pi, \Upsilon_{y}, \Sigma_{u}\right)$ reduced-form parameters and the elements in $B$ structural parameters. Moreover, we use the terms 'identification of $B$ ', 'identification of the SVAR' and 'identification of the shocks' interchangeably. Let

$$
A_{y}:=\left(\begin{array}{ccc}
\Pi_{1} & \cdots & \Pi_{k} \\
I_{n(k-1)} & 0_{n(k-1) \times n}
\end{array}\right)
$$

be the VAR companion matrix. The responses of the variables in $Y_{t+h}$ to one standard deviation structural shock $\varepsilon_{j t}$ is captured by the IRFs:

$$
I R F_{j}(h):=\left(J_{n}^{\prime}\left(A_{y}\right)^{h} J_{n}\right) b_{j}, \quad h=0,1,2, \ldots
$$

where $b_{j}$ is the $j$-th column of $B, j=1, \ldots, n$ and $J_{n}:=\left(I_{n}: 0_{n \times n(k-1)}\right)$ is a selection matrix such that $J_{n} J_{n}^{\prime}=I_{n}$. Standard local and global identification results for the SVAR in eq. (1) are reviewed in the Technical Supplement. These conditions require imposing a set of restrictions on the elements of $B$ which need not be imposed in the proxy-SVAR methodology, see below.

Let $\mathcal{F}_{t}$ be the econometrician's information set at time $t$, which will be defined below. Our first assumption postulates the correct specification of the SVAR and the nonsingularity of the matrix of structural parameters $B$, which is the only requirement we place on this matrix.

Assumption 1 (DGP) The DGP belongs to the class of models in eq. (1) which satisfy the following conditions:

(i) $E\left(u_{t} \mid \mathcal{F}_{t-1}\right)=0_{n \times 1}, E\left(u_{t} u_{t}^{\prime} \mid \mathcal{F}_{t-1}\right)=\Sigma_{u}<\infty$;

(ii) the companion matrix $A_{y}$ in eq. (2) is stable, i.e. all of its eigenvalues lie inside the unit disk;

(iii) the matrix $B$ is nonsingular.

We now consider, without loss of generality, the following partition of the vector of structural shocks:

$$
\varepsilon_{t}:=\left(\begin{array}{c}
\varepsilon_{1, t} \\
\varepsilon_{2, t}
\end{array}\right) \quad \begin{gathered}
g \times 1 \\
(n-g) \times 1
\end{gathered}
$$

where $\varepsilon_{1, t}$ is the $g \times 1$ subvector of structural shocks henceforth denoted 'instrumented structural shocks', and $\varepsilon_{2, t}$ is the $(n-g) \times 1$ subvector of other structural shocks, denoted 'non-instrumented shocks'. The instrumented structural shocks are ordered first for notational convenience only: the ordering of variables is irrelevant in this setup. We come back on these shocks below. Given the corresponding partition of reduced form VAR disturbances, $u_{t}:=\left(u_{1, t}^{\prime}, u_{2, t}^{\prime}\right)$, where $u_{1, t}$ and 
$u_{2, t}$ have the same dimensions as $\varepsilon_{1, t}$ and $\varepsilon_{2, t}$, we partition the matrix of structural parameters $B$ compatible with eq. (4), i.e.

$$
B:=\left(\begin{array}{cc}
B_{1}: & B_{2} \\
n \times g & n \times(n-g)
\end{array}\right):=\left(\begin{array}{c:c}
B_{11} & B_{12} \\
\hdashline B_{21} & B_{22}
\end{array}\right) \quad \begin{array}{cc}
g \times g & g \times(n-g) \\
\hdashline(n-g) \times g & (n-g) \times(n-g)
\end{array}
$$

where dimensions of submatrices are reported below and alongside blocks. In eq. (5), $B_{1}$ is the $n \times g$ submatrix containing the structural parameters (on-impact coefficients) associated with the instrumented shocks $\varepsilon_{1, t}$ and $B_{2}$ is the $n \times(n-g)$ submatrix containing the structural parameters associated with the non-instrumented shocks $\varepsilon_{2, t} ; \operatorname{rank}\left(B_{1}\right)=g$ and $\operatorname{rank}\left(B_{2}\right)=n-g$ because of Assumption 1(iii).

The external instruments approach postulates that given the partitions in eq.s (4)-(5), there are available $r \geq g$ 'external' (to the SVAR) variables called instruments, and collected in the $r \times 1$ vector $Z_{t}$, which can be used to identify the dynamic causal effect of $\varepsilon_{1, t}$ on $Y_{t+h}, h=0,1, \ldots$, without the need to restrict the elements in $B$. Thus, the instrumented structural shocks in $\varepsilon_{1, t}$ are the shocks of primarily interest of the analysis but, as it will be shown below, under certain conditions also the 'other' structural shocks in $\varepsilon_{2, t}$ can be indirectly identified by the instruments in $Z_{t}$. The key properties of $Z_{t}$ are formalized in the next assumption.

Assumption 2 (External instruments: relevance and validity) Given the SVAR in eq. (1) and Assumption 1, it exists a $r \times 1$ vector of observable variables $Z_{t}$ such that $r \geq g$, and:

$$
\operatorname{Cov}\left(Z_{t}, \varepsilon_{t}\right)=E\left(Z_{t} \varepsilon_{t}^{\prime}\right)=\left(\begin{array}{cc}
E\left(Z_{t} \varepsilon_{1, t}^{\prime}\right) & \vdots \\
r \times g & E\left(Z_{t} \varepsilon_{2, t}^{\prime}\right) \\
r(n-g)
\end{array}\right):=H_{\Phi}:=\left(\Phi: 0_{r \times(n-g)}\right)
$$

where the matrix $\Phi$ is $r \times g$ and is such that it has full column-rank when evaluated at its true value $\Phi_{0}$

(ii) $\operatorname{Cov}\left(Z_{t-i}, \varepsilon_{t}\right)=0_{r \times n}, i=1,2, \ldots$

(iii) $Z_{t}-E\left(Z_{t}\right)$ is covariance stationary.

Assumption 2(i) is standard in the literature on identification of SVARs through external instruments but is typically presented under the constraint $r=g$, see e.g. Stock and Watson $(2008,2012,2018)$, Mertens and $\operatorname{Ravn}(2013,2014)$. The conditions $E\left(Z_{t} \varepsilon_{1, t}^{\prime}\right)=\Phi$ and $E\left(Z_{t} \varepsilon_{2, t}^{\prime}\right)=0_{r \times(n-g)}$ in eq. (6) mirror the 'relevance' and 'exogeneity' hypotheses that characterize instrumental variable methods (Bowden and Turkington, 1984) ${ }^{3}$ The matrix

\footnotetext{
${ }^{3}$ Note that the relevance condition here involves the link between observed instruments and unobserved shocks, not observed endogeneous regressors and, moreover, the instruments do not serve to remove the correlation between endogenous regressors and disturbance terms but rather they provide additional moment conditions which allow to identify the shocks.
} 
$H_{\Phi}:=\left(\Phi: 0_{r \times(n-g)}\right)$ collects these conditions. We call the matrix $\Phi$ the 'relevance matrix' (or 'matrix of relevance parameters') throughout the paper. The condition $\operatorname{rank}\left(\Phi_{0}\right)=g$ ensures that each column of $\Phi$ carries independent - not redundant - information on the instrumented structural shocks. The case mainly treated in the literature obtains for $r=g=1$ (i.e. one instrument is used to identify a single structural shock of interest), and implies that $\Phi=\phi$ $(=\varphi)$ is a scalar and $H_{\Phi}:=\phi\left(1: 0_{1 \times(n-g)}\right)$ is a row. By considering the general case $r \geq g$, we mimick the situation that occurs in the instrumental variable regressions when the number of instruments is allowed to be larger than the number of estimated parameters.

In general, the matrix $\Phi$ in eq. (6) needs not be 'full', meaning that it might contain zeros elements. To account for this situation, we collect the non zero elements of $\Phi$ in the $c \times 1$ vector $\varphi$, and use the following parameterization of restrictions (in explicit form):

$$
\phi:=\operatorname{vec}(\Phi)=S_{\Phi} \varphi
$$

where $S_{\Phi}$ is an $r g \times c$ full column-rank selection matrix, $c \leq r g$.

Assumption 2(ii) imposes an orthogonality restrictions between lagged values of $Z_{t}$ and the structural shocks, and is consistent with the MDS property $E\left(\varepsilon_{t} \mid \mathcal{F}_{t-1}\right)=0_{n \times 1}$ implied by Assumption 1(i). It is intended that the econometrician's information set contains both the $Y_{t} \mathrm{~s}$ and the external instruments $Z_{t}$, i.e. $\sigma\left(Y_{t}, Z_{t}, \ldots, Y_{1}, Z_{1}\right) \subseteq \mathcal{F}_{t}$. Observe that Assumption 2(ii) does not imply that $Z_{t}$ must be uncorrelated with past structural shocks, i.e. in our setup it may hold the condition $\operatorname{Cov}\left(Z_{t}, \varepsilon_{t-i}\right)=E\left(Z_{t} \varepsilon_{t-i}^{\prime}\right) \neq 0_{r \times n}, i=1,2, \ldots$ Finally, Assumption 2(iii) is a stability condition consistent with the stationarity of the SVAR.

Under Assumption 2, the external instruments $Z_{t}$ provide 'additional' moment conditions which complement the standard covariance restrictions $\Sigma_{u}=B B^{\prime}$ delivered by the SVAR, and can be exploited in estimation by avoiding to impose direct restrictions on $B$. More specifically, from eq.s (4)-(5) and eq. (6) it follows that

$$
\begin{gathered}
\Sigma_{Z, u}:=\operatorname{Cov}\left(Z_{t}, u_{t}\right)=E\left(Z_{t} \varepsilon_{t}^{\prime}\right) B^{\prime} \\
=H_{\Phi} B^{\prime}=\left(\Phi: 0_{r \times(n-g)}\right)\left(\begin{array}{c}
B_{1}^{\prime} \\
B_{2}^{\prime}
\end{array}\right)=\Phi B_{1}^{\prime}=\left(\Phi B_{11}^{\prime}: \Phi B_{12}^{\prime}\right)
\end{gathered}
$$

where eq. (8) can be also written in the form

$$
\begin{array}{lc}
\Sigma_{u_{1}, Z}=B_{11} \Phi^{\prime} & g \times r \\
\Sigma_{u_{2}, Z}=B_{12} \Phi^{\prime} & (n-g) \times r
\end{array}
$$

and $\Sigma_{Z, u}=\left(\Sigma_{Z, u_{1}}: \Sigma_{Z, u_{2}}\right), \Sigma_{u_{1}, Z}=\Sigma_{Z, u_{1}}^{\prime}, \Sigma_{u_{2}, Z}=\Sigma_{Z, u_{2}}^{\prime}$. The proxy-SVAR methodologies developed independently by Stock and Watson (2012) and Mertens and Ravn (2013) are essentially based on the relationship in eq. (8) (or, equivalently, in eq. (9) ) and the restriction 
$r=g 4^{4}$ When $r=g$, the moment conditions in eq. (8) (9) can be used to estimate $B_{1}$ either without incorporating the standard covariance restrictions provided by the SVAR model, or by adding the covariance restrictions to eq. (8) and then using (generalized) method of moments techniques.

In this paper we focus on the case $r \geq g$ and cast the identification analysis and estimation of proxy-SVARs within a likelihood-based setup. In the next sections we present the general framework, identification issues and estimation methods.

\section{The augmented-constrained SVAR}

We complement the properties of the external instruments stated in Assumption 2 with a statistical auxiliary model for $Z_{t}$. The idea is to build a 'larger' system which incorporates the SVAR and the specified auxiliary model for the instruments and forms the basis of approach to proxy-SVARs.

The next assumption formalizes the features of the auxiliary model for the instruments.

Assumption 3 (External instruments: auxiliary model) Given the SVAR in eq. (1) and Assumption 1, an $r \times 1$ vector of external instruments $Z_{t}$ which satisfies Assumption 2, it is further assumed that the dynamics of $Z_{t}$ is generated by the system

$$
\begin{gathered}
Z_{t}=\Theta(L) Z_{t-1}+\Gamma(L) Y_{t-1}+\Upsilon_{z} D_{z, t}+\Upsilon_{z, y} D_{y, t}+v_{Z, t} \\
v_{Z, t}=H_{\Phi} \varepsilon_{t}+\omega_{t}=\Phi \varepsilon_{1, t}+\omega_{t}
\end{gathered}
$$

where $\Theta(L):=\Theta_{1}-\ldots-\Theta_{p} L^{p-1}$ is a matrix polynomial in the lag operator $L$ whose coefficients are in the $r \times r$ matrices $\Theta_{i}, i=1, \ldots, p ; \Gamma(L):=\Gamma_{1}-\Gamma_{2} L \ldots-\Gamma_{q} L^{q-1}$ is a matrix polynomial in the lag operator $L$ whose coefficients are in the $r \times n$ matrices $\Gamma_{j}, j=1, \ldots, q ; D_{z, t}$ is an $d_{z}$-dimensional vector containing deterministic components (constant, dummies, etc.) specific to $Z_{t}$ and not already included in $D_{y, t}$ in eq. (1); $\Upsilon_{z}$ and $\Upsilon_{z, y}$ are the $r \times d_{z}$ and $r \times d_{y}$ matrices of coefficients associated with $D_{z, t}$ and $D_{y, t}$, respectively; $v_{Z, t}:=Z_{t}-E\left(Z_{t} \mid \mathcal{F}_{t-1}\right)$ is an $r \times 1$ disturbance term with positive definite covariance matrix $\Sigma_{v}$, $\omega_{t}$ is a White Noise measurement error term uncorrelated with $\varepsilon_{t}\left(E\left(\varepsilon_{t} \omega_{t}^{\prime}\right)=0_{n \times g}\right)$ with positive definite covariance matrix $E\left(\omega_{t} \omega_{t}^{\prime}\right):=\Sigma_{\omega}<\infty$.

Assumption 3(i)-(ii) is key to our approach. The auxiliary statistical model for the external instruments is a general reduced form in which the dynamics of $Z_{t}$ depends on its own lags

\footnotetext{
${ }^{4}$ Mertens and Ravn (2013) have further noticed that the restrictions in eq.s [8]-(9) admit an IV interpretation along the lines indicated by Hausman and Taylor (1983) for simultaneous systems of equations.
} 
$Z_{t-1}, \ldots, Z_{t-p}$, the predetermined 'control' variables $Y_{t-1}, \ldots, Y_{t-k}$, and a set of (non overlapping) deterministic components in $D_{z, t}$ and $D_{y, t}$, respectively. The disturbance term $v_{Z, t}:=Z_{t}-E\left(Z_{t} \mid\right.$ $\mathcal{F}_{t-1}$ ) in eq. 10 reads as the vector of 'filtered external instruments', and eq. (11) links $v_{Z, t}$ to the structural shocks through the matrix $H_{\Phi}$ given in eq. (6). The additive measurement error $\omega_{t}$ captures the idea that the external instruments are imperfectly correlated with the instrumented structural shocks

Under Assumption 3, the covariance restrictions in eq. (6) can be reinterpreted as follows $6^{6}$

$$
\Sigma_{Z, u}:=\operatorname{Cov}\left(Z_{t}, u_{t}\right)=E\left(Z_{t} u_{t}^{\prime}\right)=E\left(v_{Z t} u_{t}^{\prime}\right)=: \Sigma_{v, u}
$$

Interestingly, under Assumptions 1-3, the SVAR in eq.s (1) and the auxiliary model for the instruments in eq.s (10)-(11) give rise to the 'larger' system:

$$
\begin{gathered}
\left(\begin{array}{c}
Y_{t} \\
Z_{t}
\end{array}\right)=\sum_{j=1}^{\ell}\left(\begin{array}{cc}
\Pi_{j} & 0_{n \times r} \\
\Gamma_{j} & \Theta_{j}
\end{array}\right)\left(\begin{array}{c}
Y_{t-j} \\
Z_{t-j}
\end{array}\right)+\left(\begin{array}{cc}
\Upsilon_{y} & 0_{n \times d_{z}} \\
\Upsilon_{z, y} & \Upsilon_{z}
\end{array}\right)\left(\begin{array}{c}
D_{y, t} \\
D_{z, t}
\end{array}\right)+\left(\begin{array}{l}
u_{t} \\
v_{Z, t}
\end{array}\right) \\
\left(\begin{array}{l}
u_{t} \\
v_{Z, t}
\end{array}\right)=\left(\begin{array}{cc}
B & 0_{n \times r} \\
H_{\Phi} & P_{\varpi}
\end{array}\right)\left(\begin{array}{c}
\varepsilon_{t} \\
\omega_{t}^{\circ}
\end{array}\right)
\end{gathered}
$$

where $\ell:=\max \{k, p, q\}, P_{\varpi}$ is the Cholesky factor of $\Sigma_{\omega}\left(\Sigma_{\omega}=P_{\varpi} P_{\varpi}^{\prime}\right)$ and $\omega_{t}^{\circ}:=P_{\varpi}^{-1} \omega_{t}$ is a normalized measurement error term. System (12) reads as an $m$-dimensional VAR, $m:=n+r$, of lag order $\ell$, which incorporates a constrained (triangular) structure in the slope coefficients, in the sense that lags of $Z_{t}$ and the deterministic variables in $D_{z, t}$ are not allowed to enter the

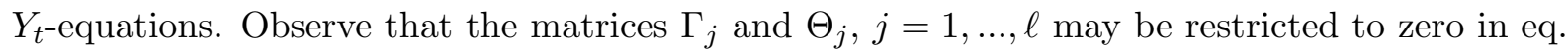
(12). System (13) maps, under certain conditions, $\xi_{t}:=\left(\varepsilon_{t}^{\prime}, \omega_{t}^{\circ \prime}\right)^{\prime}$ onto $\eta_{t}:=\left(u_{t}^{\prime}, v_{Z, t}^{\prime}\right)^{\prime}$.

We call the joint system given by eq.s (12)-(13) the 'augmented-constrained' SVAR (ACSVAR) and for future reference we compact it in the expression

$$
W_{t}=\tilde{\Psi} F_{t}+\tilde{\Upsilon} D_{t}+\eta_{t}, \eta_{t}=\tilde{G} \xi_{t}
$$

where $W_{t}:=\left(Y_{t}^{\prime}, Z_{t}^{\prime}\right)^{\prime}$ and $\eta_{t}:=\left(u_{t}^{\prime}, v_{Z, t}^{\prime}\right)^{\prime}$ are $m \times 1, \eta_{t}$ has covariance matrix $\Sigma_{\eta}:=E\left(\eta_{t} \eta_{t}^{\prime}\right)$, $F_{t}:=\left(W_{t-1}^{\prime}, \ldots, W_{t-\ell}^{\prime}\right)^{\prime}$ is $f \times 1(f=m \ell), D_{t}:=\left(D_{y, t}^{\prime}, D_{z, t}^{\prime}\right)^{\prime}$ is $d \times 1\left(d:=d_{y}+d_{z}\right)$ and, finally, $\xi_{t}:=\left(\varepsilon_{t}^{\prime}, \omega_{t}^{\circ \prime}\right)^{\prime}$ is $m \times 1$. We use the symbol ' $\sim$ ' over the matrices $\Psi$ and $\Upsilon$ and $G$ to remark that

\footnotetext{
${ }^{5}$ As it stands, system $10-11$ does not take into explicity account the possibility of censoring on the external instruments. However, censoring can be introduced by amending system 10 in many ways. For instance, as in Mertens and Ravn (2013), censoring is modelled by using dummy variables. More sophisticated censoring processes might in principle be adapted to system 11 - 10 depending on the specific problem at hand.

${ }^{6}$ To see that the equality holds, it is sufficient to post-multiply both sides of system (10) by $u_{t}^{\prime}$, take expectations and use the orthogonality condition $E\left(X_{t} u_{t}^{\prime}\right)=0_{n k \times n}$ guaranteed by Assumption 1 1 (i) and the ortogonality condition $E\left(\left(Z_{t-1}^{\prime}, \ldots, Z_{t-p}^{\prime}\right)^{\prime} u_{t}^{\prime}\right)=0_{r p \times n}$ guaranteed by Assumption 2 (ii).
} 
these incorporate particular sets of zero restrictions. The structure of the matrix $\tilde{G}$ deserves special attention:

$$
\tilde{G}:=\left(\begin{array}{c:c}
B & 0_{n \times r} \\
\hdashline H_{\Phi} & P_{\varpi}
\end{array}\right)=\left(\begin{array}{cccc}
B_{1} & B_{2} & 0_{n \times r} \\
\hdashline \Phi & 0_{r \times(n-g)} & P_{\varpi}
\end{array}\right)
$$

so that it is seen that it contains the structural parameters in $\beta:=\operatorname{vec}(B)$, the relevance parameters in $\varphi$ and the parameters in $\varpi:=\operatorname{vech}\left(P_{\varpi}\right)$. The covariance restrictions $\Sigma_{\eta}=\tilde{G} \tilde{G}^{\prime}$ implied by the AC-SVAR are therefore given by

$$
\begin{array}{ll}
\Sigma_{u}=B B^{\prime} & \text { SVAR symmetry } \\
\Sigma_{v, u}=\Phi B_{1}^{\prime} & \text { External instruments } \\
\Sigma_{v}=\Phi \Phi^{\prime}+\Sigma_{\omega} & \text { External instruments }
\end{array}
$$

and map the parameters $\beta, \varphi$ and $\varpi$ into $\Sigma_{u}, \Sigma_{v, u}$ and $\Sigma_{v}$.

We use the AC-SVAR model and the mapping in eq.s (16)-(18) to address two cases of interest. Consider the partitions in eq.s (4)-(5). One case of interest is the multiple shocks approach, where given the $r \geq g$ valid external instruments for $\varepsilon_{1, t}$, the objective is the identification of the dynamic causal effects of all $n$ structural shocks in $\varepsilon_{t}$, including the non-instrumented structural shocks in $\varepsilon_{2, t}$. This amounts to identify all columns of the matrix $B$ and to compute the IRFs in eq. (3) for $j=1, \ldots, n$. Identification and estimation issues in this case are analyzed in Section 4. The second case is the partial shock approach, where the objective of the analysis is the identification of the dynamic causal effects of the $g$ instrumented shocks in $\varepsilon_{1, t}$ alone, ignoring $\varepsilon_{2, t}$. This requires the identification of $B_{1}$ alone, and the computation of the IRFs in eq. (3) for $j=1, \ldots, g, g<n$. Identification and estimation issues in this case are analyzed in Section 5 .

Before moving to identification and estimation issues, it is worth framing the AC-SVAR framework in the current literature on proxy-SVARs. In the 'frequentist' literature, only PlagborgMøller and Wolf (2018) make explicit use of an auxiliary model for external instruments to make inference on forecast variance decompositions. They refer to a specification which resembles the one in system (10)-11) where the dynamics is based on infinite lags.7 In the Bayesian framework, Caldara and Herbst (2018) use a specification similar to the one in eq.s (12)-(13) based on $r=g=1$ (hence $Z_{t}$ and $\varepsilon_{1, t}$ are scalars), where the parameters $\Gamma_{j}$ s and $\Theta_{j}$ s are set to zero 8 Caldara and Herbst (2018) observe that the likelihood described by the joint system for

\footnotetext{
${ }^{7}$ Olea et al. (2016) observe in their footnote 4 that a simple example of a statistical model for the instruments is given by the measurement error model:

$$
Z_{t}=f\left(\varepsilon_{1, t}\right)+a_{1} Y_{t-1}+\ldots+a_{p} Y_{t-p}+v_{t}
$$

where the measurement error $v_{t}$ is a stationary process independent of $\varepsilon_{t}$. However, they do not exploit explicitly this model.

${ }^{8}$ Actually, their SVAR parameterization is such that the matrix $B^{-1}$ pre-multiplies both sides of system 1 .
} 
$W_{t}:=\left(Y_{t}^{\prime}, Z_{t}^{\prime}\right)^{\prime}$ offers a coherent modeling of all sources of uncertainty and hence, allows the proxy to inform the estimation of both reduced-form and structural parameters; see also Arias et al. (2018).

Finally, it is worth remarking that the AC-SVAR model is substantially different from the 'VAR-X approach' to proxy-SVARs put forth in Paul (2017). In Paul (2017), the instrument(s) $Z_{t}$ enter directly the SVAR model for $Y_{t}$ in the form of 'exogenous' (X) variable(s), and the coefficients associated with these variables capture directly the impact of the shocks of interest on the variables. In our setup, the $Y_{t}$-equations of the AC-SVAR model are not affected at all by the external instrument(s) and are connected to $Z_{t}$ solely through the covariance matrix $\Sigma_{u, v}$; conversely, the dynamics of the external instruments may depend on $Y_{t-1}, \ldots, Y_{t-p}$.

\section{Multiple shocks approach}

In this case, we use the instruments $Z_{t}$ for $\varepsilon_{1, t}$ to identify the dynamic responses of $Y_{t+h}$ to all structural shocks in $\varepsilon_{t}$, including also the ones in $\varepsilon_{2, t}$. It is therefore necessary to estimate the whole matrix $B$ in order to compute the IFRs in eq. (3) for $j=1, \ldots, n$. Section 4.1 deals with identification issues and Section 4.2 with the estimation.

\subsection{Identification}

Since the matrix $B$ is contained in $\tilde{G}$ in eq. 15 , the identification of the system can be based on the AC-SVAR model in eq. (14) and 'standard' methods. For instance, as the matrix $\tilde{G}$ in eq. (15) features homogeneous restrictions, separable across columns, one can check whether the model satisfies the sufficient conditions for global identification in Rubio-Ramirez et al. (2011) on a case-by-case basis 9 General necessary and sufficient conditions for identification can also be provided, as shown next.

Let $G$ be the unrestricted version of the matrix $\tilde{G}$ in eq. 15 (i.e. before any restriction is imposed). Then, $\tilde{G}$ is the counterpart of $G$ which satisfies the constraints (in explicit form)

$$
\operatorname{vec}(G)=S_{\tilde{G}} \theta
$$

where $\theta:=\left(\beta^{\prime}, \varphi^{\prime}, \varpi^{\prime}\right)^{\prime}=\left(\operatorname{vec}\left(B_{1}\right)^{\prime}, \operatorname{vec}\left(B_{2}\right)^{\prime}, \varphi^{\prime}, \varpi^{\prime}\right)^{\prime}$ and $S_{G}$ is an $m^{2} \times a_{\tilde{G}}$ full column-rank selection matrix. The next proposition provides the necessary and sufficient rank conditions for (local) identification and the necessary order conditions.

Proposition 1 Given the SVAR in eq. (1), an $r \times 1$ vector $Z_{t}$ of external instruments and Assumptions 1,3, consider the identification of the shocks in $\varepsilon_{t}$, i.e. the IRFs in eq. (3)

\footnotetext{
${ }^{9} \mathrm{~A}$ concrete example is reported in the Technical Supplement.
} 
for $j=1, \ldots, n$. Let $\theta_{0}$ be the true value of $\theta:=\left(\beta^{\prime}, \varphi^{\prime}, \varpi^{\prime}\right)^{\prime}$, where $\theta$ has dimension $a_{\tilde{G}} \times 1$, $a_{\tilde{G}}:=n^{2}+c+\frac{1}{2} r(r+1)$. Then:

(i) necessary and sufficient condition for identification is:

$$
\operatorname{rank}\left\{D_{m}^{+}\left(\tilde{G}_{0} \otimes I_{m}\right) S_{\tilde{G}}\right\}=a_{\tilde{G}}
$$

where $\tilde{G}_{0}$ is the matrix in eq. 15 evaluated in a neighbourhood of $\theta_{0}$ and is 'regular', $S_{G}$ is the $m^{2} \times a_{\tilde{G}}$ full column-rank selection matrix given in eq. 19);

(ii) necessary order condition for identification is $a_{\tilde{G}} \leq \frac{1}{2} m(m+1)$ or, equivalently $n^{2}+c \leq$ $\frac{1}{2} n(n+1)+n r$.

Proof: Technical Supplement.

Two remarks are in order. First, in the common empirical case in which a single instrument is used to identify a single structural shock, $r=g=1, \Phi=\phi=\varphi$ is a scalar, $c=1, B_{1}:=b_{1}$ is an $n \times 1$ column and $B_{2}$ is an $n \times(n-1)$ sub-matrix obtained from $B$ by deleting its first column. In this case, the necessary order condition in Proposition 1 (1.ii) suggests that the identification of the $n$ columns of the matrix $B:=\left(b_{1}: B_{2}\right)$ is at most possible for bi-variate SVARs $(n=2)$ because the inequality $n^{2}+1 \leq \frac{1}{2} n(n+1)+n$ does not hold for $n \geq 3$. The interpretation is that a single external instrument is not generally sufficient to identify more than two shocks. Second, according to Proposition 1, when the AC-SVAR is overidentified, the system features $l:=\frac{1}{2} m(m+1)-a_{\tilde{G}}$ testable restrictions which can be interpreted as a specification test for the SVAR with external instruments, see the next section.

\subsection{Estimation}

The estimation of the AC-SVAR model in eq. 114 with $\tilde{G}$ restricted as in eq. 15 follows, under the conditions of Proposition 1, 'standard' methods. To do so it is however necessary to impose the zero restrictions which characterize the matrices $\tilde{\Psi}$ and $\tilde{\Upsilon}$ in estimation. The specification the reduced form system behind the AC-SVAR model can be based on the following strategy:

Step-1 Given $Z_{t}$, estimate an $m$-dimensional VAR system for $W_{t}:=\left(Y_{t}^{\prime}, Z_{t}^{\prime}\right)^{\prime}$, and use standard methods to determine the VAR lag order $\ell:=\ell^{o p}$, where $\ell^{o p} \geq k$;

Step-2 Determined $\ell:=\ell^{o p}$ in the previous step, re-estimate the VAR for $W_{t}:=\left(Y_{t}^{\prime}, Z_{t}^{\prime}\right)^{\prime}$ with $\ell^{o p}$ lags, by imposing the set of zero restrictions (triangular structures) that characterize $\tilde{\Psi}$ and $\tilde{\Upsilon}$. This can be done, under the auxiliary assumption of Gaussian disturbances, by constrained maximum likelihood 10

\footnotetext{
${ }^{10}$ Which is asymptotically equivalent to an iterated version of a (Feasible) GLS estimator, see Lutkepol (1993). In other words, OLS are not efficient in this setup as OLS require unrestricted $\Psi$ and $\Upsilon$.
} 
Defined $\sigma_{\eta}^{+}:=\operatorname{vech}\left(\Sigma_{\eta}\right)$, the (concentrated) log-likelihood of the reduced form system can be written in the form

$$
L_{T}\left(\sigma_{\eta}^{+}\right):=\frac{-T m}{2} \log (2 \pi)-\frac{T}{2} \log \left(\operatorname{det}\left(\Sigma_{\eta}\right)\right)-\frac{T}{2} \operatorname{tr}\left(\Sigma_{\eta}^{-1} \hat{\Sigma}_{\eta}\right)
$$

where $\hat{\Sigma}_{\eta}:=(1 / T) \sum_{t=1}^{T} \hat{\eta}_{t} \hat{\eta}_{t}^{\prime}, \hat{\eta}_{t}:=\left(W_{t}-\widehat{\tilde{\Psi}} F_{t}-\widehat{\tilde{\Upsilon}} D_{t}\right), t=1, \ldots, T$, and $\widehat{\tilde{\Psi}}$ and $\widehat{\tilde{\Upsilon}}$ are the Gaussian maximum likelihood estimates of $\tilde{\Psi}$ and $\tilde{\Upsilon}$, respectively. Note that the log-likelihood in eq. 21) is concentrated with respect to the constrained parameters in $(\tilde{\Psi}, \tilde{\Upsilon})$. Assuming that the rank condition in eq. 20 of Proposition 1 holds, the log-likelihood of the AC-SVAR is obtained form eq. 21 by imposing the restriction $\Sigma_{\eta}:=\Sigma_{\eta}(\theta)=\tilde{G}(\theta) \tilde{G}(\theta)^{\prime}$ and reads:

$$
L_{T}^{s}(\theta):=L_{T}\left(\sigma_{\eta}^{+}(\theta)\right):=\frac{-T m}{2} \log (2 \pi)-\frac{T}{2} \log \left(\operatorname{det}\left(\Sigma_{\eta}(\theta)\right)\right)-\frac{T}{2} \operatorname{tr}\left(\Sigma_{\eta}^{-1}(\theta) \hat{\Sigma}_{\eta}\right) .
$$

The maximization of $L_{T}^{s}(\theta)$ can be performed by standard methods discussed e.g. in Amisano and Giannini (1997) 11 Under Assumptions 1, 3 , the estimator $\hat{\theta}_{T}:=\max _{\theta} L_{T}^{s}(\theta)$ is consistent and asymptotically Gaussian.

When the AC-SVAR model satisfies the rank condition of Proposition 1 (or the sufficient global identification conditions in Rubio-Ramirez et al. (2011)) and is overidentified, it is possible to compute the LR test:

$$
L R_{T}:=-2\left(L_{T}^{s}\left(\hat{\theta}_{T}\right)-L_{T}\left(\hat{\sigma}_{\eta}^{+}\right)\right)
$$

which is distributed asymptotically as a $\chi^{2}(l)$ variable with $l:=\frac{1}{2} m(m+1)-a_{\tilde{G}}$ degrees of freedom. In the (G)IV (GMM) framework, when the number of instruments (moment conditions) is larger than the number of estimated parameters, it is possible to compute Sargan's specification test (Hansen's J-test), which is typically interpreted as a specification test for the estimated model. In this framework, the LR test in eq. 23) can be interpreted similarly, i.e. as a specification test for the empirical validity of the estimated proxy-SVAR. The $(2,2)$ block of the matrix $\tilde{G}$ in the right-hand side of eq. 15 incorporates $r(n-g)$ zero restrictions implied by the exogeneity condition assumed for the external instruments (i.e. the matrix $H_{\Phi}$ is part of the matrix $\tilde{G}$ ). When the exogeneity condition is violated in the data generating process, the $L R_{T}$ test in eq. (23) rejects the overidentification restrictions. We investigate the finite sample performance of the $L R_{T}$ test when the exogeneity of the instruments is wrongly assumed in a set of Monte Carlo experiments reported in the Technical Supplement to save space.

In case of exact identification, or if the overidentification restrictions are not rejected by the LR test, the IRFs are estimated from eq. (3) by replacing $A_{y}$ with the maximum likelihood

\footnotetext{
${ }^{11}$ In practice, estimation can be carried out by adpating any existing econometric package which features SVARs. To do so, however, it is necessary to suitably restrict the estimation of the coefficients $(\tilde{\Psi}, \tilde{\Upsilon})$ as implied by the structure of the AC-SVAR model.
} 
estimate $\hat{A}_{y}$ derived from the AC-SVAR model, and $b_{j}$ with the corresponding elements taken from the $j$-th column of $\widehat{\tilde{G}}=\tilde{G}\left(\hat{\theta}_{T}\right)$, for $j=1, \ldots, n$. Asymptotic and/or bootstrap confidence intervals for the IRFs can be computed along the lines discussed in e.g. Lütkepohl and Kilian (2017, Ch. 14).

\section{$5 \quad$ Partial shock approach}

In this case, we use $Z_{t}$ for $\varepsilon_{1, t}$ to identify the dynamic responses of $Y_{t+h}$ to the structural shocks in $\varepsilon_{1, t}$ alone, i.e. there is a perfect match between the instrumented shocks and the structural shocks of interest. It is therefore necessary to estimate $B_{1}$ in order to compute the IFRs in eq. (3) for $j=1, \ldots, g<n$. Section 5.1 deals with identification issues and Section 5.2 with the estimation.

\section{$5.1 \quad$ Identification}

The analysis is based on the mapping in eq.s (16)-(17). The difficulty with the moment conditions in eq. 16 is that the covariance restrictions $\Sigma_{u}=B B^{\prime}=B_{1} B_{1}^{\prime}+B_{2} B_{2}^{\prime}$ involve also the parameters in $B_{2}$ which are not of interest, and need to be marginalized out. To get rid of $B_{2}$, we use the invertibility of $\Sigma_{u}=B B^{\prime}$ (Assumption 1) and the transformation:

$$
\begin{gathered}
\Sigma_{Z, u} \Sigma_{u}^{-1} \Sigma_{u, Z}=\Phi B_{1}^{\prime}\left(B B^{\prime}\right)^{-1} B_{1} \Phi^{\prime}=\Phi B_{1}^{\prime}\left(B^{\prime}\right)^{-1} B^{-1} B_{1} \Phi^{\prime} \\
=\Phi B_{1}^{\prime}\left(B^{\prime}\right)^{-1} B^{-1} B_{1} \Phi^{\prime}=\Phi \Phi^{\prime}
\end{gathered}
$$

where eq. 24 has been derived by using the fact that the condition $B^{-1} B=I_{n}=\left(B^{-1} B_{1}\right.$ : $\left.B^{-1} B_{2}\right)$ implies

$$
B^{-1} B_{1}=\left(\begin{array}{c}
I_{g} \\
0_{(n-g) \times g}
\end{array}\right), \quad B^{-1} B_{2}=\left(\begin{array}{c}
0_{g \times(n-g)} \\
I_{(n-g)}
\end{array}\right) .
$$

Eq. (24) is re-written as

$$
\Xi=\Phi \Phi^{\prime}
$$

where $\Xi=\Sigma_{v, u} \Sigma_{u}^{-1} \Sigma_{u, v}$ is an $r \times r$ symmetric positive semi-definite matrix, and forms, along with eq. 17) which is here reported for convenience:

$$
\Sigma_{v, u}=\Phi B_{1}^{\prime}
$$

a set of moment conditions which allow to identify $B_{1}$ and $\Phi$.

Let $\zeta:=\left(\operatorname{vech}(\Xi)^{\prime}, \operatorname{vec}\left(\Sigma_{v, u}\right)^{\prime}\right)^{\prime}$ be the $a \times 1$ vector of coefficients which appear in the lefthand-side of eq.s (26)-27), $a:=1 / 2 r(r+1)+n r$. It is seen that $\zeta$ is a nonlinear function of the 
reduced form parameters in $\sigma_{\eta}^{+}:=\operatorname{vech}\left(\Sigma_{\eta}\right)$. Let $\vartheta:=\left(\alpha^{\prime}, \varphi^{\prime}\right)^{\prime}$ be the $(e+c) \times 1$ vector containing the 'free' (unconstrained) parameters which enter the matrices $B_{1}$ and $\Phi$ in eq.s (26)-27). Even if in principle we want to estimate $B_{1}$ unrestrictedly, we allow for the possibility of constraints on its elements, covering cases which might be of economic interest, see the empirical illustration in Section 6. Thus, we collect the $e$ unrestricted (non-zero) elements of $\beta_{1}:=\operatorname{vec}\left(B_{1}\right)$ in the vector $\alpha(e \leq n g)$ and model these constraints by using the explicit form $\beta_{1}=S_{B_{1}} \alpha$, where $S_{B_{1}}$ is an $n g \times e$ full-column rank selection matrix. Obviously, when all elements of $\beta_{1}$ are unrestricted, $S_{B_{1}}=I_{n g}$ and $e=n g$. As is already known, the $c$ non-zero elements contained in the relevance matrix $\Phi$ are collected in the vector $\varphi(c \leq r g)$, see eq. (7). In light of these definitions, the moment conditions in eq.s 26)-(27) can be simplified in the expression

$$
\zeta=g(\vartheta)
$$

where $g(\vartheta):=\left(\operatorname{vech}\left(\Phi \Phi^{\prime}\right)^{\prime}, \operatorname{vec}\left(\Phi B_{1}^{\prime}\right)^{\prime}\right)^{\prime}$ is a nonlinear differentiable vector function. Eq. 28) defines a distance between $\zeta$ and $\vartheta$. By applying standard matrix derivatives rules, the associated Jacobian matrix $\digamma_{\vartheta}:=\partial \zeta / \partial \vartheta^{\prime}$ is $a \times(e+c)$ and has the following structure:

$$
\digamma_{\vartheta}:=\left(\begin{array}{c:c}
0_{1 / 2 r(r+1) \times n g} & D_{r}^{+}\left(\Phi \otimes I_{r}\right) \\
\hdashline\left(I_{n} \otimes \Phi\right) K_{n g} & \left(B_{1} \otimes I_{r}\right)
\end{array}\right)\left(\begin{array}{c:c}
S_{B_{1}} & 0_{n g \times c} \\
\hdashline 0_{r g \times e} & S_{\Phi}
\end{array}\right) .
$$

We have proved the proposition that follows. 12

Proposition 2 Given the SVAR in eq. (1) and Assumptions 1, 3, consider the identification of the shocks in $\varepsilon_{1, t}$, i.e. the IRFs in eq. (3) for $j=1, \ldots, g<n$. Let $B_{1}$ be the $n \times g$ submatrix of $B:=\left(B_{1}: B_{2}\right)$, and assume that $\beta_{1}:=\operatorname{vec}\left(B_{1}\right)$ satisfies the restrictions $\beta_{1}=S_{B_{1}} \alpha$, where $\alpha$ is the $e \times 1$ vector $(e \leq n g)$ of unrestricted elements and $S_{B_{1}}$ is an $n g \times e$ selection matrix of full column-rank. Let $\vartheta_{0}$ be the true value of $\vartheta:=\left(\alpha^{\prime}, \varphi^{\prime}\right)^{\prime}$, where $\vartheta$ has dimension $(e+c) \times 1$. Then:

(i) necessary and sufficient condition for identification is

$$
\operatorname{rank}\left(\digamma_{\vartheta_{0}}\right)=e+c
$$

where $\digamma_{\vartheta_{0}}$ is the matrix $\digamma_{\vartheta}$ in eq. (29) evaluated in a neighbourhood of $\vartheta_{0}$, and is 'regular'; (ii) necessary order condition for identification is $c+e \leq 1 / 2 r(r+1)+n r$.

\footnotetext{
${ }^{12}$ As it happens for the necessary and sufficient rank condition in Proposition 1, which is valid up to sign changes in the columns of the matrix $\tilde{G}$, also in this case, the parameters in $\Phi$ and $B_{1}$ are identified up to sign normalization, in the sense that if a given matrix $\tilde{\Phi}$ satisfies $\Xi=\tilde{\Phi} \tilde{\Phi}^{\prime}$, also the matrix $\tilde{\Phi}^{*} \neq \tilde{\Phi}$, obtained from $\tilde{\Phi}$ by simply changing the sign of one of its columns, will satisfy $\Xi=\tilde{\Phi}^{*} \tilde{\Phi}^{* \prime}$. The moment conditions involving the matrix $\Sigma_{v}$ (the covariance matrix of the filtered external instruments) are not necessary to derive the result in Proposition 2. It is sufficient to observe that given $\Sigma_{v}$ and $\Phi$, the covariance matrix of measurement errors $\Sigma_{\omega}$ is automatically determined.
} 
According to Proposition 2, the proxy-SVAR is identified (and hence is testable) when $e+c<$ $\frac{1}{2} r(r+1)+n r$. In the typical case treated in the literature based on one external instrument and and one structural shock of interest, $r=g=1, \Phi=\phi=\varphi$ is a scalar $\left(c=1, S_{\Phi}:=1\right), D_{r}^{+}=1$, $K_{n 1}=I_{n}$, and assuming that there are no restrictions on $B_{1}=\beta_{1}\left(S_{B_{1}}:=I_{n}\right)$ the proxy SVAR is exactly identified. The Jacobian $\digamma_{\vartheta}$ in eq. $(29)$ collapses to the $(n+1) \times(n+1)$

$$
\digamma_{\vartheta}:=\left(\begin{array}{cccc:c}
0 & 0 & \cdots & 0 & \phi \\
\hdashline & \phi I_{n} & & & \beta_{1}
\end{array}\right)
$$

which shows that the condition $\phi_{0} \neq 0$ (Assumption 2(ii)) is necessary and sufficient for the proxy-SVAR to be identified 13

\subsection{Estimation}

Provided the necessary and sufficient conditions in Proposition 2 are satisfied, the estimation of $\vartheta:=\left(\alpha^{\prime}, \varphi^{\prime}\right)^{\prime}$ can based on the minimization of the 'distance' in eq. [28), given a consistent estimate of $\zeta$, where it is seen that $\zeta$ is a function of the reduced form parameters in $\sigma_{\eta}^{+}:=$ $\operatorname{vech}\left(\Sigma_{\eta}\right)$. Thus, in order to obtain $\zeta$, it is necessary to preliminarily estimate the reduced form model associated with the AC-SVAR system. The the specification steps 1-2 discussed in Section 4.2 are still useful to this purpose.

In Appendix B, we show that under Assumptions 1-3, the (indirect) maximum likelihood estimator of $\zeta, \hat{\zeta}_{T}=h\left(\hat{\sigma}_{\eta}^{+}\right)$, is such that

$$
T^{1 / 2}\left(\hat{\zeta}_{T}-\zeta_{0}\right) \rightarrow_{d} N\left(0_{a \times 1}, \Omega_{\zeta}\right), \Omega_{\zeta}:=\digamma_{\lambda} \Omega_{\lambda} \digamma_{\lambda}^{\prime}
$$

where $\zeta_{0}:=h\left(\sigma_{\eta, 0}^{+}\right)$is the true value of $\zeta, h(\cdot)$ is a differentiable vector function and the Jacobian matrix $\digamma_{\lambda}$ and the covariance matrix $\Omega_{\lambda}$ depend on the covariance parameters in $\sigma_{\eta}^{+}$, and can be estimated consistently given $\hat{\sigma}_{\eta, T}^{+}:=\operatorname{vech}\left(\hat{\Sigma}_{\eta}\right)$.

Starting from the result in eq. (30) and given the distance in eq. (28), it seems 'natural' to estimate $\vartheta$ by the CMD problem:

$$
\min _{\vartheta}\left(\hat{\zeta}_{T}-g(\vartheta)\right)^{\prime} \hat{\Omega}_{\zeta}^{-1}\left(\hat{\zeta}_{T}-g(\vartheta)\right)
$$

where $Q(\vartheta):=\left(\hat{\zeta}_{T}-g(\vartheta)\right)^{\prime} \hat{\Omega}_{\zeta}^{-1}\left(\hat{\zeta}_{T}-g(\vartheta)\right)$ is a 'weighted' distance function. Under Assumptions 1.3, the optimization problem in eq. (31) delivers a consistent asymptotically Gaussian estimator

\footnotetext{
${ }^{13}$ The structure of this Jacobian matrix shows clearly that if the relevance parameter, evaluated at its true value, satisfies the local-to-zero embedding: $\phi_{0}:=T^{1 / 2} \varrho$, where $\varrho \neq 0$ is a constant, the proxy-SVAR is not identified asymptotically. See Olea et al. (2016) and Lunsford (2015) for robust inferential procedures in these cases.
} 
of $\vartheta$, i.e.

$$
T^{1 / 2}\left(\hat{\vartheta}_{T}-\vartheta_{0}\right) \rightarrow_{d} N\left(0_{(e+c) \times 1}, \Omega_{\vartheta}\right), \Omega_{\vartheta}:=\left(\digamma_{\vartheta} \Omega_{\zeta}^{-1} \digamma_{\vartheta}^{\prime}\right)^{-1}
$$

where the Jacobian matrix $\digamma_{\vartheta}$ is given in eq. (29) and can be estimated consistently by replacing $B_{1}(\alpha)$ and $\Phi(\varphi)$ with the estimates taken from $\hat{\vartheta}_{T}:=\left(\hat{\alpha}_{T}^{\prime}, \hat{\varphi}_{T}^{\prime}\right)^{\prime}$.

When according to Proposition 2 the model is overidentified, the estimation of $\vartheta$ from eq. (31) delivers automatically a test of overidentifying restrictions because, under the null hypothesis $\zeta_{0}=g\left(\vartheta_{0}\right)$, the quantity $T Q\left(\hat{\vartheta}_{T}\right)$ converges asymptotically to a $\chi^{2}(l)$ random variable with $l:=1 / 2 r(r+1)+n r-(e+c)$ degree of freedoms. Our Monte Carlo experiments (reported in the Technical Supplement to save space) confirm that also in this case the test $T Q\left(\hat{\vartheta}_{T}\right)$ rejects the overidentification restrictions when the external instruments are erroneously assumed valid.

In case of exact identification, or when the overidentification restrictions are not rejected by the test, the IRFs of interest in eq. (3) can be estimated by replacing $A_{y}$ with $\hat{A}_{y}$ derived from the AC-SVAR model, and $b_{j}$ with the $j$-th column of $\hat{B}_{1}$, for $j=1, . ., g$, where $\hat{B}_{1}$ is reconstructed from $\hat{\beta}_{1, T}:=S_{B_{1}} \hat{\alpha}_{T}$. Also in this case, (asymptotic and/or bootstrap) confidence intervals for the IRFs can be computed by using standard methods, see e.g. Lütkepohl and Kilian (2017, Ch. 14).

\section{The response of uncertainty to real economic activity shocks}

In this section, we apply our identification and likelihood-based approach to proxy-SVARs to analyze empirically whether measures of macroeconomic and financial uncertainty, taken from Jurado et al. (2015) and Ludvigson et al. (2018), respectively, respond on-impact (other than with lags) to real economic activity shocks. This analysis finds its root in a recent debate on the role of uncertainty in the business cycle which attends to establish whether uncertainty is better approximated as an exogenous source of decline in real economic activity, or rather as an endogenous response to first-moment shocks; see Ludvigson et al. (2018) for an excellent review ${ }^{14}$ Following the characterization in Ludvigson et al. (2018), uncertainty is considered 'exogenous' if it does not respond on-impact (instantaneously) to first-moment shocks (it might respond, however, with lags), and is considered 'endogenous' otherwise. This issue has been

\footnotetext{
${ }^{14} \mathrm{An}$ established view is that uncertainty is recessionary in presence of real options effects (Bloom, 2009), precautionary saving and/or financial frictions (e.g. Christiano, Motto and Rostagno, 2014), and that its effects can be amplified in extreme conditions like high financial stress (e.g. Alfaro et al., 2016; Arellano et al., 2012; Gilchrist et al., 2014), or the zero lower bound (Basu and Bundick, 2017). Nevertheless, uncertainty appears also to endogenously increase during recessions, as lower economic growth induces greater dispersion at the micro level and higher aggregate volatility, and this induces one to consider uncertainty 'endogenous', i.e. a consequence, not a cause, of declining economic activity.
} 
investigated by small-scale non-recursive SVARs in Ludvigson et al. (2018), Angelini et al. (2017) and Carriero et al. (2018), using different methods to identify the shocks. These authors report robust empirical evidence on the exogeneity of financial uncertainty, but mixed empirical evidence on the exogeneity of macroeconomic uncertainty.

We consider a SVAR for $Y_{t}:=\left(a_{t}, U_{M, t}, U_{F, t}\right)^{\prime}$, including real economic activity, $a_{t}$, proxied by the growth rate of the $\log$ of the industrial production index, $a_{t}:=\Delta i p_{t}$, (source Fred), a measure of macroeconomic uncertainty, $U_{M, t}$, and a measure of financial uncertainty $U_{F, t}$. In particular, $U_{M t}$ is a measure of 1-month ahead macroeconomic uncertainty taken from Jurado et al. (2015), and $U_{F t}$ is a measure of 1-month ahead financial uncertainty and is taken from Ludvigson et al. (2018) 15 The estimation sample covers the period 2008M1-2015M4 ( $T=88$ monthly observations) that we term the 'Great Recession + Slow Recovery' period 16 This period is particularly informative to infer whether uncertainty measures respond on-impact to real economic activity shock. Indeed, the Great Recession + Slow Recovery period broadly coincides with the zero lower bound constraint on the short-term nominal interest rate. The argument contends that the zero lower bound and the implied restricted the ability of the central bank to stabilize the economy by 'standard' interventions are possible mechanisms of 'endogenous' uncertainty. In particular, given the difficulty by the central bank to offset negative shocks during the zero lower bound, economic agents expect large dispersion in future economic growth, see Plante et al. (2018) and Basu and Bundick (2015). According to this view, $U_{M t}$ and $U_{F t}$ are expected to respond on-impact, not only with lags, to a real economic activity shock.

The specified reduced form VAR for $Y_{t}:=\left(a_{t}, U_{M, t}, U_{F, t}\right)^{\prime}$ includes a constant and $k=4$ lags. The SVAR is based on $u_{t}=B \varepsilon_{t}$, where $u_{t}:=\left(u_{a, t}, u_{M, t}, u_{F, t}\right)^{\prime}$ is the vector of VAR reduced form disturbances and $\varepsilon_{t}:=\left(\varepsilon_{a, t}, \varepsilon_{M, t}, \varepsilon_{F, t}\right)^{\prime}$ is the vector of structural shocks, where $\varepsilon_{a, t}$ is the real economic activity shock we are interested in and for which we employ external instruments (hence $g=1)$. Thus, we have in mind the partitions $\varepsilon_{t}:=\left(\varepsilon_{1, t}, \varepsilon_{2, t}\right)^{\prime}$, where $\varepsilon_{1, t}:=\left(\varepsilon_{a, t}\right)$ and $\varepsilon_{2, t}:=\left(\varepsilon_{M, t}, \varepsilon_{F, t}\right)$, and $B:=\left(B_{1}: B_{2}\right)$, where $B_{1}$ is $3 \times 1\left(\beta_{1}:=\operatorname{vec}\left(B_{1}\right)=B_{1}\right)$ and $B_{2}$ is $3 \times 2$. We are primarily concerned with the identification of the parameters in $\beta_{1}:=\left(b_{1, a}, b_{2, a}, b_{3, a}\right)^{\prime}$ which capture the instantaneous effects of $\varepsilon_{a, t}$ on $Y_{t}$ and, more in general, allow to track the dynamic causal response of $Y_{t+h}$ to $\varepsilon_{a, t}$. Our objective is also testing the hypothesis $b_{21}=0, b_{31}=0$ of no instantaneous (on-impact) response of $U_{M, t}$ and $U_{F, t}$ to $\varepsilon_{a, t}$.

\footnotetext{
${ }^{15}$ These uncertainty measures are estimated as the average of the time-varying volatility, as produced by stochastic volatility models, of the forecast error of each series in a large panel of macroeconomic $\left(U_{M t}\right)$ and financial variables $\left(U_{F t}\right)$, conditional on information available.

${ }^{16}$ We do not consider the entire period 1960-2015 as it is done in e.g. Ludvigson et al. (2018), because the empirical evidence reported in Angelini et al. (2017) suggests that the unconditional VAR covariance matrix of $Y_{t}:=\left(a_{t}, U_{M, t}, U_{F, t}\right)^{\prime}, \Sigma_{u}$, is subject to at least two structural breaks during the period 1960-2015.
} 
Imagine for the moment that one valid external instrument $Z_{t}$ is used for $\varepsilon_{a, t}$, i.e. $r=$ $g=c=1$ and $\Phi=\phi=\varphi$. According to Proposition 1, no other shock other than $\varepsilon_{a, t}$ can be identified as it is not satisfy the necessary identification order condition. According to Proposition $2, \vartheta:=\left(\beta_{1}^{\prime}, \varphi\right)^{\prime}=\left(b_{1, a}, b_{2, a}, b_{3, a}, \varphi\right)^{\prime}$, and conditional on the validity of the rank condition, we have an exactly identified proxy-SVAR $(c+e=1+3=1 / 2 r(r+1)+n r=1+3)$. Furthermore, adding the restrictions $b_{2, a}=0, b_{3, a}=0$ implies 2 testable over-identification restrictions $(c+e=1+1<1 / 2 r(r+1)+n r=1+3)$. Observe that in this case, the matrix $\tilde{G}$ in eq. 15 takes the form

$$
\tilde{G}:=\left(\begin{array}{ccc:c}
B_{1} & B_{2} & 0_{n \times r} \\
\hdashline \Phi & 0_{r \times(n-g)} & P_{\varpi}
\end{array}\right)=\left(\begin{array}{cccc:c}
b_{1, a} & b_{1, M} & b_{1, F} & 0 \\
b_{2, a} & b_{2, M} & b_{2, F} & 0 \\
b_{3, a} & b_{3, M} & b_{3, F} & 0 \\
\hdashline \varphi & 0 & 0 & \sigma_{\varpi}
\end{array}\right)
$$

where $\sigma_{\varpi}^{2} \equiv \Sigma_{\varpi}$, so that it is clearly seen that there is not enough information to identify $B_{2}$ other than $B_{1}$. Consider now the case in which $r=2>g$ valid instruments are used for $\varepsilon_{a, t}$, so that the matrix $\tilde{G}$ in eq. 15 now reads

$$
\tilde{G}:=\left(\begin{array}{c:ccc}
B_{1} & B_{2} & 0_{n \times r} \\
\hdashline \Phi & 0_{r \times(n-g)} & P_{\varpi}
\end{array}\right)=\left(\begin{array}{cccc:cc}
b_{1, a} & b_{1, M} & b_{1, F} & 0 & 0 \\
b_{2, a} & b_{2, M} & b_{2, F} & 0 & 0 \\
b_{3, a} & b_{3, M} & b_{3, F} & 0 & 0 \\
\varphi_{1} & 0 & 0 & p_{1,1}^{\varpi} & 0 \\
\varphi_{2} & 0 & 0 & p_{2,1}^{\varpi} & p_{2,2}^{\varpi}
\end{array}\right)
$$

and $\varphi_{1}:=E\left(v_{Z_{1}, t} \varepsilon_{a, t}\right)$ and $\varphi_{2}:=E\left(v_{Z_{2}, t} \varepsilon_{a, t}\right)$ are the relevance parameters, and the $p_{i, j}^{\varpi}$ are the elements of the Cholesky factor of $\Sigma_{\varpi}$. It is seen that also in this case the necessary order condition of Proposition 1 fails and there is not enough information to identify $B_{2}$ other than $B_{1}{ }^{17}$ According to Proposition 2, conditional on the validity of the rank condition, the proxy-SVAR is overidentified with $1 / 2 r(r+1)+n r-(c+e)=3+6-(2+3)=4$ testable overidentification restrictions even when $\beta_{1}$ is left unrestricted. Imposing the further restrictions $b_{2, a}=0, b_{3, a}=0(c=2, e=1)$ leads to 6 overidentification restrictions.

Based on these considerations, we decided to exploit two valid external instruments to identify the shock $\varepsilon_{a, t}(r=2>g)$ in the AC-SVAR model, which amounts to identify the first

\footnotetext{
${ }^{17}$ In the Technical Supplement we show that adding e.g. a further instrument in the system, say $Z_{3 t}$, which is correlated with the macroeconomic uncertainty shock $\varepsilon_{M, t}$ and is uncorrelated with the financial uncertainty shocks $\varepsilon_{F, t}$ and the real economic activity shock $\varepsilon_{a, t}$, leads to an AC-SVAR model for which the necessary and sufficient identification conditions of Proposition 1 are satisfied and hence it is possible to identify also the effects of the macroeconomic and uncertainty shocks in a multiple shocks approach.
} 
column of $\tilde{G}$ in eq. 33 . The two external instruments are constructed as follows. Let hour $s_{t}$ be the log of hours worked and $c a p_{t}$ the log of the index of total capacity utilization (source FRED database). We then consider the linear regression model

$$
Z_{t}^{*}=\delta_{0}+\delta_{1} \text { Gold }_{t}+R_{t}, t=1, \ldots, T
$$

where $Z_{t}^{*}:=\left(\Delta \text { hour }_{t}, \Delta \text { cap }_{t}\right)^{\prime}$ and Gold is the 'price of gold' variable build in Piffer and Podstawski (2018) as a proxy of uncertainty shocks. In this regression, $\delta_{0}$ is an $r \times 1$ constant, $\delta_{1}$ is an $r \times 1$ vector of slope parameters and $R_{t}$ is the vector of disturbances. We estimate the regression model in eq. (34) by OLS on the period 2008M1-2015M4 and take the residuals $\hat{R}_{t}$ as external instruments for the real economic activity shock, i.e. $Z_{t}=\hat{R}_{t}=\left(Z_{1, t}, Z_{2, t}\right)^{\prime}$, $t=1, \ldots, T$. These two variables are by construction expected to be correlated with the real economic activity shock, and are expected to be 'orthogonal' to Gold $t_{t}$ and therefore, following the arguments in Piffer and Podstawski (2018), they should not reflect any significant correlations with the uncertainty shocks confined in $\varepsilon_{2, t}:=\left(\varepsilon_{M, t}, \varepsilon_{F, t}\right)^{\prime}$.

Given these two instruments, and following the specification steps 1-2 in Section 4.2, we estimate an $m$-dimensional $(m:=n+r=5)$ reduced form system for $W_{t}:=\left(Y_{t}^{\prime}, Z_{t}\right)^{\prime}:=$ $\left(a_{t}, U_{M, t}, U_{F, t}, Z_{1, t}, Z_{2, t}\right)^{\prime}$, including a constant. This provides the reduced form of our ACSVAR model. The specification analysis lead to a system based on $\ell:=\ell^{o p}=4$ lags, where in particular the $Y_{t}$-equations do not depend on $Z_{t}$ as they correspond to those of the original SVAR model (as implied by the structure of system (14)), while the two $Z_{t}$-equations of the system depend on two lags of $Z_{t}(p=2)$ and four lags of $Y_{t}$.

The upper panel of Table 1 summarizes the maximum likelihood estimates of the reduced form covariance matrix $\Sigma_{\eta}$, with associated standard errors (main diagonal and upper triangle) and the implied correlations (lower triangle). (We do not report the estimated autoregressive coefficients in $\tilde{\Psi}$ and those in $\tilde{\Upsilon}$ to save space). The correlations which are significant at the $5 \%$ level of significance are denoted with asterisks. Table 1 also summarizes the value of the maximized log-likelihood function, see eq. (21), and some residuals diagnostic tests. The diagnostic tests show that the disturbances of the estimated model are uncorrelated and non-Gaussian and, notably, are not affected by ARCH-type components.

The correlations estimated in Table 1 provide some indirect prima facie reduced form evidence about the relevance and the exogeneity of the selected instruments: it can be noticed that the instrument $Z_{1 t}$ ( $\Delta$ hour $s_{t}$ 'purged' from Gold $)_{t}$ ) is significantly correlated with the disturbance $\hat{u}_{a, t}(0.41)$ and is not significantly correlated with the disturbances $\hat{u}_{M, t}$ and $\hat{u}_{F, t}(0.09$ and 0.06 , respectively); likewise, the instrument $Z_{2 t}\left(\Delta \text { cap }_{t} \text { 'purged' from Gold }\right)_{t}$ is significantly correlated with the disturbance $\hat{u}_{a, t}(0.76)$ and is not significantly correlated with the disturbances $\hat{u}_{M, t}$ and $\hat{u}_{F, t}(-0.05$ and 0.12 , respectively). 
The lower panel of Table 1, left side, reports the CMD estimates of the parameters $\vartheta:=$ $\left(\alpha^{\prime}, \varphi\right)^{\prime}$ obtained by the partial shock approach discussed in Section 5.2, see eq. (31). In this case the elements of $\beta_{1}$ are left unrestricted, hence $\beta_{1}=\operatorname{vec}\left(B_{1}\right)=\alpha\left(S_{B_{1}}:=I_{3}\right)$. The estimated $\hat{\vartheta}_{T}:=\left(\hat{b}_{1, a, T}, \hat{b}_{2, a, T}, \hat{b}_{3, a, T}, \hat{\varphi}_{T}^{\prime}\right)^{\prime}$ is reported in the table with associated standard errors. It is seen that while the estimated relevance parameters $\varphi_{1}$ and $\varphi_{2}$ are strongly significant, the estimated coefficients $b_{2, a}$ and $b_{3, a}$, which capture the on-impact effect of the real economic activity shock on macroeconomic and financial uncertainty have negative sign (as expected) but are not significant at the $5 \%$ nominal level of significance. From the estimates of $\varphi_{1}$ and $\varphi_{2}$, we derive the following correlations related to the relevance condition: $\widehat{\operatorname{Corr}}\left(Z_{1 t}, \varepsilon_{a, t}\right)=$ $0.156 /(0.13)^{1 / 2}=0.43^{*}$ and $\widehat{\operatorname{Corr}}\left(Z_{2 t}, \varepsilon_{a, t}\right)=0.422 /(0.29)^{1 / 2}=0.78^{*}$. The test statistic $T Q\left(\hat{\vartheta}_{T}\right)$ for the 4 overidentification restrictions, reported in Table 1 below estimated parameters, is equal to 2.39 and has an (asymptotic) p-value of 0.81 which lends ample support to the estimated proxy-SVAR.

Next, we move to the right side of the lower panel of Table 1 . In this case, the parameters in $\beta_{1}$ are estimated by imposing the restrictions $b_{2, a}=0, b_{3, a}=0$ of no response of macroeconomic and financial uncertainty to the identified real economic activity shock. In practice, $\beta_{1}$ is restricted as $\beta_{1}=S_{B_{1}} \alpha$, where $S_{B_{1}}^{\prime}:=(1,0,0)$ and the parameter $\alpha:=b_{1, a}$ captures the on-impact effect of one-standard deviation real economic activity shock on industrial production growth; we use the notation $\hat{\vartheta}_{T}^{c}:=\left(\hat{\alpha}_{T}^{\prime} S_{B_{1}}^{\prime}, \hat{\varphi}_{T}^{\prime}\right)^{\prime}$ to indicate the estimates obtained in this case from the CMD problem in eq. (31). The restrictions $b_{2, a}=0, b_{3, a}=0$ are tested by computing a LR-type test statistic constructed as $T Q\left(\hat{\vartheta}_{T}^{c}\right)-T Q\left(\hat{\vartheta}_{T}\right)$, where $T Q\left(\hat{\vartheta}_{T}\right)$ refers to the model estimated with $\beta_{1}$ unrestricted (left-bottom side of Table 1$)$ and $T Q\left(\hat{\vartheta}_{T}^{c}\right)$ refers to the model estimated with $b_{2, a}=0, b_{3, a}=0$ imposed (right-bottom side of Table 1 ). The test statistic is equal to 2.14 and has an (asymptotic) p-value of 0.34 which permits to accept the hypothesis that the chosen measures of macroeconomic and financial uncertainty do not respond on-impact to the real economic activity shock.

The IRFs are are plotted in Figure 1. These are estimated under the null $b_{2, a}=0, b_{3, a}=0$, therefore they are obtained from eq. (3) by replacing $A_{y}$ with the maximum likelihood estimate $\hat{A}_{y}$ derived from the AC-SVAR model, and $B_{1}=\beta_{1}=b_{1}$ with $\hat{\beta}_{1, T}^{c}:=S_{B_{1}} \hat{\alpha}_{T}$. The graphs track the dynamic responses of $U_{M, t+h}$ and $U_{F, t+h}$ to one-standard deviation real economic activity shock for $h=0,1,2, \ldots, h_{\max }=60$ months ( 5 years). Considering the results in Brüggemann et al. (2016) and the absence of ARCH-type components in the reduced form disturbances detected in the upper panel of Table 1, the $90 \%$ bootstrap confidence intervals for the IRFs reported in Figure 1 are computed through an iid nonparametric bootstrap procedure.

Figure 1 shows that given a one-standard deviation real economic activity shock, (i) macroe- 
conomic and financial uncertainty do not respond significantly on-impact (as already seen from Table 1); (ii) macroeconomic and financial uncertainty decline significantly one month after the shock; (3) the effect of the real economic activity shock is absorbed within two periods (months), in the sense that the responses of macroeconomic and financial uncertainties are not significant for $h=2,3 \ldots$.

Overall, the results discussed in this empirical illustration rule out the hypothesis that macroeconomic and financial uncertainty respond on-impact to a real economic activity shock on the Great Recession+Slow Recovery period. This evidence confirms the results in Angelini et al. (2017), who identify the structural shocks by using the identification-through-heteroskedasticity approach developed in Bacchiocchi and Fanelli (2015) and Bacchiocchi et al. (2018). It also lines up with Carriero et al. (2018), who identify the structural shocks by using a stochastic volatility approach (using both monthly and quarterly variables) but using measures of macroeconomic and financial uncertainty one at a time. Carriero et al. (2018) report mild evidence for the endogeneity of uncertainty, and this evidence is limited to financial uncertainty and, mainly, to monthly variables. Finally, our empirical results are partially consistent with Ludvigson et al. (2018), who report that sharply higher uncertainty about real economic activity in recessions is an endogenous response to other shocks, while uncertainty about financial markets is a likely source of the fluctuations. Ludvigson et al. (2018) focus on the period 1960-2015 and identify the structural shocks through a set-identification approach where direct constraints are imposed on the magnitude of the structural shocks in correspondence of some extraordinary events such as e.g. the 'Black Monday' of October 1987, or the 2007-2009 financial crisis.

\section{Concluding remarks}

We have provided general identification results for proxy-SVARs for the case in which $r$ external instruments are used for $g \geq 1$ structural shocks of interest. In the multiple shocks approach, the identification and maximum likelihood estimation of proxy-SVARs can be analyzed by using standard methods with minor adaptations. In the partial shock approach, the identification analysis and estimation of proxy-SVARs can be framed in a classical minimum distance approach, where maximum likelihood estimation is used to obtain consistent estimates of a set of reduced form coefficients. In both approaches, overidentified proxy-SVARs can be easily tested against the data.

The usefulness of the suggested approach has been illustrated empirically by analyzing whether commonly employed measures of macroeconomic and financial uncertainty respond on-impact to real economic activity shocks in the U.S. after the Global Financial Crisis. In 
particular, we have used two external instruments to identify the real economic activity shock and tested the empirical validity of the so-obtained overidentified proxy-SVAR. Empirical results suggest that both macroeconomic and financial uncertainty respond significantly to the identified real economic activity shock one month after the shock, not on-impact. This evidence enriches an ongoing empirical debate on the role of uncertainty in the business cycle.

\section{Appendix A: proof of Proposition 1}

Part 1.(i) The proof follows from a straightforward application of the reults in eq.s (TS.2)-(TS.3) of the Technical Supplement to the the matrix

$$
\tilde{G}:=\left(\begin{array}{c:c}
B & 0_{n \times g} \\
\hdashline H_{\Phi} & P_{\varpi}
\end{array}\right)=\left(\begin{array}{ccc:c}
B_{1} & B_{2} & 0_{n \times r} \\
\hdashline \Phi & 0_{r \times(n-g)} & P_{\varpi}
\end{array}\right) .
$$

In particular, define the $m^{2} \times 1$ vector $\gamma:=\left(\operatorname{vec}\left(B_{1}\right)^{\prime}, \operatorname{vec}\left(B_{2}\right)^{\prime}, \operatorname{vec}(\Phi)^{\prime}, \operatorname{vec}\left(0_{r \times(n-g)}\right)^{\prime}, \operatorname{vec}\left(0_{n \times r}\right)^{\prime}, \operatorname{vec}\left(P_{\varpi}\right)^{\prime}\right)^{\prime}$ which contains the same information as $\operatorname{vec}(\tilde{G})$ but in a different order. It is seen that the linear relationship between $\gamma$ and $\theta:=\left(\beta^{\prime}, \varphi^{\prime}, \varpi^{\prime}\right)^{\prime} \equiv\left(\beta_{1}^{\prime}, \beta_{2}^{\prime}, \varphi^{\prime}, \varpi^{\prime}\right)^{\prime}$ is given by

$$
\gamma=\left(\begin{array}{cccc}
I_{n g} & 0_{n g \times n(n-g)} & 0_{n g \times c} & 0_{n g \times \frac{1}{2} r(r+1)} \\
0_{n(n-g) \times n g} & I_{n(n-g)} & 0_{n(n-g) \times c} & 0_{n(n-g) \times \frac{1}{2} r(r+1)} \\
0_{r^{2} \times n g} & 0_{r^{2} \times n(n-g)} & S_{\Phi} & 0_{r^{2} \times \frac{1}{2} r(r+1)} \\
0_{r(n-g) \times n g} & 0_{r(n-g) \times n(n-g)} & 0_{g r(n-g) \times c} & 0_{r(n-g) \times \frac{1}{2} r(r+1)} \\
0_{r g \times n g} & 0_{n r \times n(n-g)} & 0_{n r \times c} & 0_{n r \times \frac{1}{2} r(r+1)} \\
0_{r^{2} \times n g} & 0_{r^{2} \times n(n-g)} & 0_{r^{2} \times c} & D_{r}
\end{array}\right) \theta
$$

which we simply in the expression $\gamma=\Omega \theta$. Then, introduce the $m^{2} \times m^{2}$ permutation matrix $\mathcal{P}$ such that:

$$
\operatorname{vec}(\tilde{G})=\mathcal{P} \gamma
$$

where it is seen that the matrix $\mathcal{P}$ applied to $\gamma$ returns $\operatorname{vec}(\tilde{G})$. By pre-multiplying both sides of eq. 35 by $\mathcal{P}$ yields the linear restrictions

$$
\operatorname{vec}(\tilde{G})=\mathcal{P} \gamma=\mathcal{P} \Omega \theta=S_{\tilde{G}} \theta
$$

where the selection matrix $S_{\tilde{G}}$ is expressed as $S_{\tilde{G}}:=\mathcal{P} \Omega$ (note that $s_{\tilde{G}}=0_{m^{2} \times 1}$ in this setup). The result is thus obtained.

Part 1.(ii). The necessary order condition follows from the fact that the matrix $D_{m}^{+}\left(\tilde{G}_{0} \otimes\right.$ $\left.I_{m}\right) S_{\tilde{G}}$ is $\frac{1}{2} m(m+1) \times a_{\tilde{G}}$, where $a_{\tilde{G}}:=\operatorname{dim}(\theta)=n^{2}+c+\frac{1}{2} r(r+1)$. 


\section{Appendix B: the asymptotic distribution of $\hat{\zeta}_{T}$}

In this Appendix we discuss in detail the result used in eq. (30) of the paper. The starting point is the asymptotic normality of the maximum likelihood estimator of the reduced form parameters of the AC-SVAR model under Assumption 1. 3. More precisely, let $\sigma_{\eta, 0}^{+}$be the true value of $\sigma_{\eta}^{+}:=\operatorname{vech}\left(\Sigma_{\eta}\right)$; then, from the (Gaussian) maximum likelihood estimation of the reduced form of the AC-SVAR model, and for $T \rightarrow \infty$,

$$
T^{1 / 2}\left(\hat{\sigma}_{\eta, T}^{+}-\sigma_{\eta, 0}^{+}\right) \rightarrow_{d} N\left(0_{1 / 2 m(m+1) \times 1}, \Omega_{\eta}\right), \Omega_{\eta}:=2 D_{m}^{+}\left(\Sigma_{\eta} \otimes \Sigma_{\eta}\right)\left(D_{m}^{+}\right)^{\prime}
$$

where $\hat{\sigma}_{\eta, T}^{+}$is derived either from the maximization of the log-likelihood in eq. 21. The asymptotic covariance matrix $\Omega_{\eta}$ can be estimated consistently by $\hat{\Omega}_{\eta, T}:=2 D_{m}^{+}\left(\hat{\Sigma}_{\eta, T} \otimes \hat{\Sigma}_{\eta, T}\right)\left(D_{m}^{+}\right)^{\prime}$.

Now define now the $1 / 2 m(m+1) \times 1$ vector $\lambda:=\left(\operatorname{vech}\left(\Sigma_{u}\right)^{\prime}, \operatorname{vec}\left(\Sigma_{v, u}\right)^{\prime}, \operatorname{vech}\left(\Sigma_{v}\right)^{\prime}\right)^{\prime}$, which contains the same elements as $\sigma_{\eta}^{+}$disposed in different order, so that $\lambda=\mathcal{P}_{\sigma} \sigma_{\eta}^{+}$, where $\mathcal{P}_{\sigma}$ is a permutation matrix. Given eq. (36) and Assumptions 1,3.

$$
T^{1 / 2}\left(\hat{\lambda}_{T}-\lambda_{0}\right) \rightarrow_{d} N\left(0_{1 / 2 m(m+1) \times 1}, \Omega_{\lambda}\right), \Omega_{\lambda}:=\mathcal{P}_{\sigma} \Omega_{\eta} \mathcal{P}_{\sigma}^{\prime}
$$

where $\hat{\lambda}_{T}:=\mathcal{P}_{\sigma} \hat{\sigma}_{\eta, T}^{+}, \lambda_{0}:=\mathcal{P}_{\sigma} \sigma_{\eta, 0}^{+}$and $\Omega_{\lambda}$ can be estimated consistently by $\hat{\Omega}_{\lambda, T}:=\mathcal{P}_{\sigma} \hat{\Omega}_{\eta, T} \mathcal{P}_{\sigma}^{\prime}$. The $a \times 1$ vector $\zeta:=\left(\operatorname{vech}(\Xi)^{\prime}, \operatorname{vec}\left(\Sigma_{v, u}\right)\right)^{\prime}$ in eq. 28$)$ depends on $\lambda$, i.e. $\zeta=h(\lambda)$, where $h(\cdot)$ is a differentiable vector function. Thus, $\hat{\zeta}_{T}=h\left(\hat{\lambda}_{T}\right), \zeta_{0}=h\left(\lambda_{0}\right)$ and from eq. 37) and the delta-method, $\hat{\zeta}_{T}$ is a maximum likelihood estimator and is asymptotically Gaussian with covariance matrix $\Omega_{\zeta}:=\digamma_{\lambda} \Omega_{\lambda} \digamma_{\lambda}^{\prime}$, where the $a \times 1 / 2 m(m+1)$ Jacobian matrix $\digamma_{\lambda}:=\partial \zeta / \partial \lambda^{\prime}$ is given by:

$$
\digamma_{\lambda}:=\left(\begin{array}{c:c:c}
D_{r}^{+}\left(\Sigma_{v, u} \Sigma_{u}^{-1} \otimes \Sigma_{v, u} \Sigma_{u}^{-1}\right) D_{n}^{+\prime} & D_{r}^{+}\left(\Sigma_{v, u} \Sigma_{u}^{-1} \otimes I_{r}\right) & 0_{1 / 2 r(r+1) \times 1 / 2 r(r+1)} \\
\hdashline 0_{n r \times 1 / 2 n(n+1)} & I_{n r} & 0_{n r \times 1 / 2 r(r+1)}
\end{array}\right) .
$$

\section{References}

Alfaro, I.N., Bloom, N. and Lin, X. (2016), The finance-uncertainty multiplier, Working Paper, Stanford University and Ohio State University.

Arias, J. E., Rubio-Ramirez, J. F. and Waggoner, D. F. (2018), Inference in Bayesian SVARs identified with external instruments, Working Paper, Federal Reserve.

Amisano, G. and Giannini, C. (1997). Topics in Structural VAR Econometrics, 2nd edn, Springer, Berlin. 
Angelini, G., Bacchiocchi, E., Caggiano, G., and Fanelli, L. (2017), Uncertainty across volatility regimes, Bank of Finland research Discussion Paper 35/2017.

Arellano, C., Bai, Y. and Kehoe, P. (2012), Financial markets and fluctuations in uncertainty, Federal Reserve Bank of Minneapolis Staff Report, March.

Bacchiocchi, E. and Fanelli, L. (2015), Identification in Structural Vector Autoregressive models with structural changes, with an application to U.S. monetary policy, Oxford Bulletin of Economics and Statistics 77, 761-779.

Bacchiocchi, E., Castelnuovo, E. and Fanelli, L. (2018), Give me a break ! Identification and estimation of the macroeconomic effects of monetary policy shocks in the US, Macroeconomic Dynamics, forthcoming.

Basu, S. and Bundik, B. (2015), Endogenous volatility at the Zero Lower Bound: Implications for stabilization policy, NBER Working Paper No. 21838

Basu, S. and Bundik, B. (2017), Uncertainty shocks in a model of effective demand, Econometrica, forthcoming.

Bowden, R.J. and Turkington, D.A. (1984), Instrumental variables, Cambridge University Press.

Bloom, N. (2009), The impact of uncertainty shocks, Econometrica 77, 623-685.

Braum, R. and Brüggemann, R. (2017), Identification of SVAR models by combining sign restrictions with external instruments, Working Paper Series 2017-07, Universiry of Konstanz.

Brüggemann, R., Jentsch, C. and Trenkler, C. (2016), Inference in VARs with conditional volatility of unknown form, Journal of Econometrics 191, 69-85.

Caggiano, G., E. Castelnuovo, and G. Pellegrino, (2017), Estimating the real effects of uncertainty shocks at the Zero Lower Bound, European Economic Review 100, 257-272.

Caldara, D. and Herbst, E. (2018), Monetary policy, real activity, and credit spreads: evidence from Bayesian proxy SVARs, American Economic Journal: Macroeconomics, forthcoming.

Caldara, D. and Kamps, C. (2017), The analytics of SVARs: A unified framework to measure fiscal multipliers, Review of Economic Studies 84, 1015-1040. 
Carriero, A., Mumatz, H., Theodoritis, K. and Theophilopoulou, A. (2015), The impact of uncertainty shocks under measurement error: A proxy SVAR approach, Journal of Money, Credit, and Banking 47, 1223-1238.

Carriero, A., Clark, T. E. and Marcellino, M. (2018), Endogenous uncertainty?, Federal Reserve Bank of Cleveland, Working Paper No. 18-05.

Carsten, J. and Lunsford, K.G. (2016), Proxy SVARs: Asymptotic theory, bootstrap inference, and the effects of income tax changes in the United States, Federal Reserve Bank of Cleveland Working Paper, No. 16-19.

Christriano, J.C., Motto, R. and Rostagno, M. (2014), Risk shocks, American Economic Review 104, 27-65.

Hamilton, J. D. (2003), What is an oil shock?, Journal of Econometrics, 113, 363-98.

Gertler, M. and Karadi, P. (2015), Monetary policy surprises, credit costs, and economic activity, American Economic Journal: Macroeconomics 7, 44-76.

Hausman, J.A. and Taylor, W.E. (1983), Identification in linear simultaneous equations models with covariance restrictions: an instrumental variables interpretation, Econometrica 51, 1527-1549.

Jentsch, C. and Lunsford, K.C. (2016), Proxy VARs: Asymptotic theory, bootstrap inference and the effects of income tax changes in the united states, Federal Reserve Bank of Cleveland, Working Paper No. 16-19.

Jurado, K., Ludvigson, S.C. and Ng, S. (2015), Measuring uncertainty, American Economic Review 105(3), 1177-1216.

Kilian, L. (2008), The economic effects of energy price shocks, Journal of Economic Literature $46,871-909$.

Leduc, S. and Liu, Z. (2016), Uncertainty shocks are aggregate demand shocks, Journal of Monetary Economics 82, 20-35.

Ludvigson, S.C., Ma, S. and Ng, S. (2017), Shock restricted Structural Vector-Autoregressions, Working Paper, version March 1, 2017.

Ludvigson, S.C., Ma, S. and Ng, S. (2018), Uncertainty and business cycles: exogenous impulse or endogenous response? Working Paper, draft dated February 15, 2018. 
Lunsford, K. G. (2015), Identifying Structural VARs with a proxy variable and a test for a weak proxy, Federal Reserve Bank of Cleveland, Working Paper No. 15-28.

Lütkepohl, H. (1993), Introduction to Multiple Time Series Analysis, Second edition, SpringerVerlag.

Lütkepohl, H. and Kilian, L. (2017), Structural Vector Autoregressive Analysis, Cambridge University Press.

Magnus, J.R. and Neudecker, H. (1999), Matrix differential calculus with applications in Statistics and Econometrics, Wiley \& Sons, Mertens, K. and Ravn, M. (2013), 2nd edition.

Mertens, K. and Ravn, M. (2012), Empirical evidence on the aggregate effects of anticipated and unanticipated U.S. Tax Policy Shocks, American Economic Journal: Economic Policy $3,145-181$.

Mertens, K. and Ravn, M. (2013), The dynamic effects of personal and corporate income tax changes in the United States, American Economic Review 103, 1212-1247.

Mertens, K. and Ravn, M. (2014), A reconciliation of SVAR and narrative estimates of tax multipliers, Journal of Monetary Economics 68, S1-S19.

Nakata, T. (2017), Uncertainty at the zero lower bound, American Economic Journal: Macroeconomics $9,186-221$.

Olea, J.L.M., Stock, J.H. and Watson, M.W. (2016), Inference in SVARs identified with an external instrument, Working Paper.

Paul, P. (2018), The time-varying effect of monetary policy on asset prices, Federal Reserve Bank of San Francisco, Working Paper No. 2017-09.

Piffer, M. and M. Podstawski (2018), Identifying uncertainty shocks using the price of gold, The Economic Journal 121, forthcoming.

Plante, M., Richter, W.A. and Throckmorton, N.A. (2018), The Zero Lower Bound and endogenous uncertainty, The Economic Journal, forthcoming.

Ramey, V. (2016), Macroeconomic shocks and their propagation, Handbook of Macroeconomics Vol. 2, 71-162

Rubio-Ramírez, J. F., Waggoner, D.F. and Zha, T. (2010), Structural Vector Autoregressions and algorithms for inference, Review of Economic Studies 77, 665-696. 
Stock, J.H (2008), What's new in Econometrics: Time Series, Lecture 7, NBER Summer Institute, available at http://www.nber.org/minicourse_2008.html

Stock, J.H and Watson, M.W. (2012), Disentangling the channels of the 2007-2009 recession, Brooking Panel of Economic Activity, Conference Paper March 22-23.

Stock, J.H. and Watson, M.W. (2018), Identification and estimation of dynamic causal effects in macroeconomics using external instruments, Economic Journal, forthcoming. 
Table 1. Estimated AC-SVAR

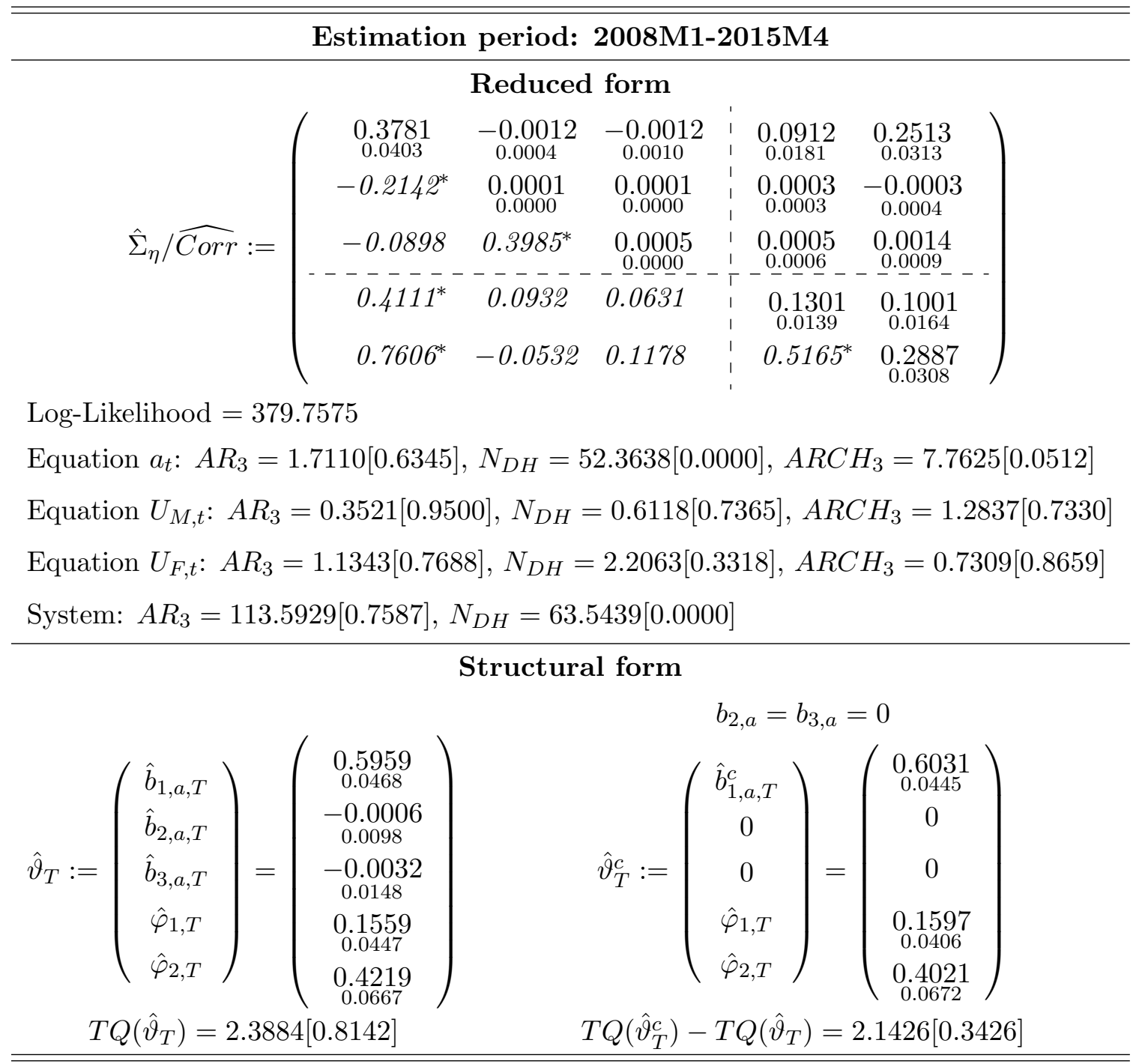

NOTES: Upper panel: Maximum Likelihood estimates of $\Sigma_{\eta}$ with associated standard errors and some diagnostic tests (the numbers in italics are the correlations and asterisks '*' denote the statistically significance at $5 \%$ level). ' $A R_{3}$ ' is an LM-type test for the absence of residual autocorrelation against the alternative of autocorrelated VAR disturbances up to 3 lags; ' $N_{H D}$ ' is the Doornik-Hansen multivariate (univariate) test for Gaussian disturbances; ' $\mathrm{ARCH}_{3}$ ' is the Engle's ARCH-type test for the absence of conditional heteroskedasticity up to 3 lags. Numbers in brackets are $p$-values. 'Log-Likelihood' is the value of the loglikelihood function in eq. (21). Lower panel: estimates of $\vartheta:=\left(\beta_{1}^{\prime}, \varphi\right)^{\prime}$ with associated standard errors. ' $T Q\left(\hat{\vartheta}_{T}\right)$ ' is the test statistic for the over-identification restriction and ' $T Q\left(\hat{\vartheta}_{T}^{c}\right)-T Q\left(\hat{\vartheta}_{T}\right)$ ' is the test statistic for $b_{2, a}=0$ and $b_{3, a}=0$, where $Q\left(\hat{\vartheta}_{T}\right)$ is in eq. (31). 


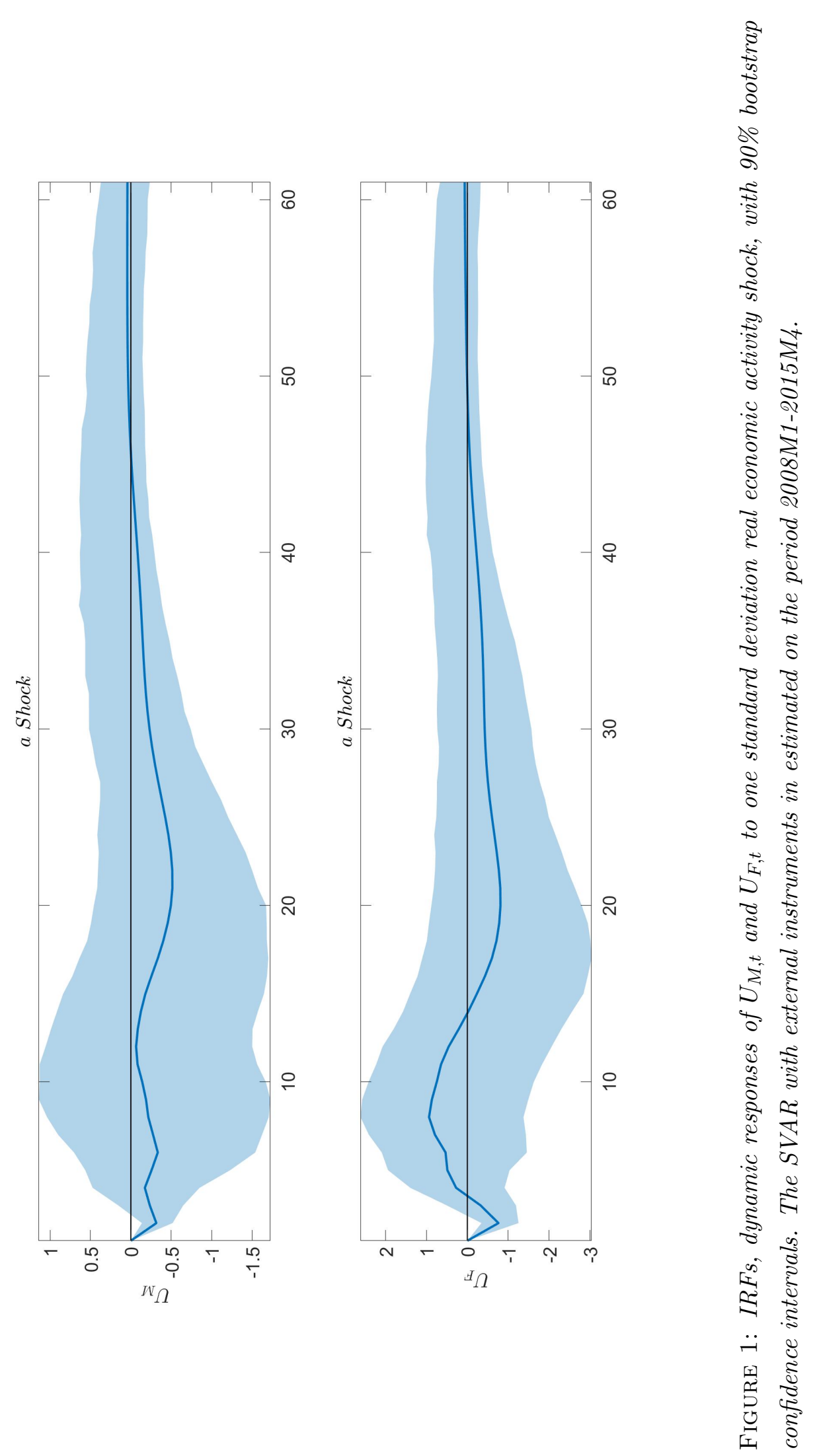




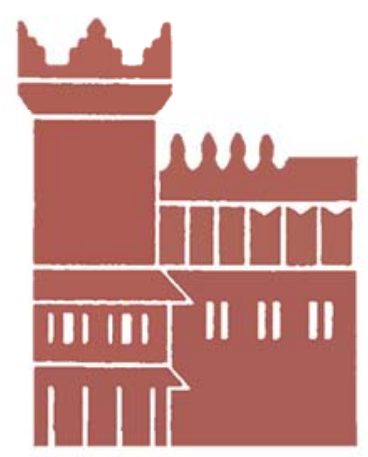

Alma Mater Studiorum - Università di Bologna DEPARTMENT OF ECONOMICS

Strada Maggiore 45

40125 Bologna - Italy

Tel. +39051 2092604

Fax +390512092664

http://www.dse.unibo.it 